TECHNISCHE UNIVERSITEIT EINDHOVEN

Faculteit Wiskunde en Informatica

Memorandum COSOR 91-28

A Compensation Approach for Two-dimensional Markov Processes

I.J.B.F. Adan

J. Wessels

W.H.M. Zijm

Eindhoven University of Technology

Department of Mathematics and Computing Science

P.O. Box 513

5600 MB Eindhoven

The Netherlands

Eindhoven, October 1991

The Netherlands 


\title{
A Compensation Approach for Two-dimensional Markov Processes
}

\author{
I.J.B.F. Adan* J. Wessels ${ }^{\dagger} \quad$ W.H.M Zijm ${ }^{\ddagger}$
}

October 26, 1991

\begin{abstract}
Several queueing processes may be modelled as random walks on a multi-dimensional grid. In this paper the equilibrium distribution for the case of a two-dimensional grid is considered. In previous research it has been shown that for some two-dimensional random walks the equilibrium distribution has the form of an infinite series of products of powers which can be constructed with a compensation procedure. The object of the present paper is to investigate under which conditions such an elegant solution exists and may be found with a compensation approach. The conditions can be easily formulated in terms of the random behaviour in the inner area and the drift on the boundaries.
\end{abstract}

\section{Introduction}

Many queueing problems may be formulated as random walks on multi-dimensional grids. There are several results on the equilibrium distributions for such random walks. Wellknown are the conditions for getting an equilibrium distribution with product form (see for instance the paper of Baskett et al. [7]). For selected problems results have been obtained using generating function techniques. Well-known in this category are the papers of Kingman [20] and of Flatto and McKean [15] on the symmetric shortest queue problem. In these papers it is shown that the generating function for the equilibrium distribution of the lengths of the two queues is meromorphic. This result can be exploited for showing that the equilibrium probabilities can be written as an infinite linear combination of product forms. However, this approach does not lead to a tractable road for finding the explicit expressions apart from the first two terms which require already considerable effort. A similar approach has been used by Hofri for a multiprogramming queueing problem with two queues involved (see Hofri [17] and also Adan et al. [6] for some additional information).

There are more general results regarding the use of generating function techniques for random walks on two-dimensional grids which are relevant for queueing problems. The work of Iasnogorodski and Fayolle [12], [13], [19] and also of Cohen and Boxma [11] shows how the analysis can be reduced to the solution of a Riemann-Hilbert boundary value

\footnotetext{
*Technical University Eindhoven, Department of Mathematics and Computing Sciences, Eindhoven, The Netherlands

†Technical University Eindhoven and International Institute for Applied Systems Analysis, Laxenburg, Austria

IUniversity Twente, Department of Mechanical Engineering, Enschede, The Netherlands
} 
problem. However, the approach does not lead to explicit expressions for the equilibrium probabilities, even not for selected problems. There is also a numerically oriented method for multi-dimensional exponential queueing systems in the form of a power series method (see Hooghiemstra et al. [18]). Blanc ([8], [9]) reports numerically satisfactory results for the shortest queue problem with up to 25 parallel queues. The method lacks a theoretical foundation and no error bounds are provided.

In a couple of recent papers ([3], [1]) the present authors have shown that the symmetric shortest queue problem and Hofri's multiprogramming problem can be treated quite elegantly by using a compensation approach. In fact, the authors have exploited the same ideas to solve the asymmetric shortest queue problem and the shortest queue problem with threshold jockeying ([4], [5]). In each case the essence of the approach is to find a sufficiently rich class of product form solutions for the equilibrium equations away from the boundaries and use this class for the construction of a linear combination of product forms which also satisfies the boundary conditions. In three of the four cases this construction is of a compensation type. This implies that, after introducing the main term, product forms are added one by one in order to compensate for the error introduced by the preceding term on one of the boundaries. Of course, this approach only works if the successive terms converge sufficiently fast to zero. In all these cases a fast numerical method is obtained which provides the user with error bounds.

The goal of the present paper is to investigate whether it is possible to generalise this compensation approach as described in the last paragraph. Therefore, we will consider a continuous-time random walk on the two-dimensional grid of the positive quadrant and try to find under which conditions the compensation approach works. In order to keep the boundary conditions simple, we consider random walks with transitions to adjoining gridpoints only (horizontally, vertically and diagonally). Of course it is required that the random walk is ergodic. The other main condition that will appear is that for transitions starting in the inner region the sum of the coordinates is not increasing. However, it also appears that the approach as described above should be amended in the following way. Depending on the boundary conditions it is possible that more than one initial product form exists, each generating a series of compensating terms. Hence, the equilibrium probabilities are represented by a linear combination of series of product forms rather than just by one series of product forms. Moreover, as also encountered in the analysis of Hofri's multiprogramming problem [1], we may have to cope with the fact that the series-representation for the solution diverges near the origin.

The paper is organised as follows. In the next section the model is introduced together with the equilibrium equations to be solved. In Section 3 the compensation method is introduced as a formal method leading to a formal solution. In Section 4 convergence conditions are investigated. Section 5 is devoted to the existence of feasible initial terms. Section 6 presents and proves the main result and, finally, Section 7 contains comments and conclusions. 


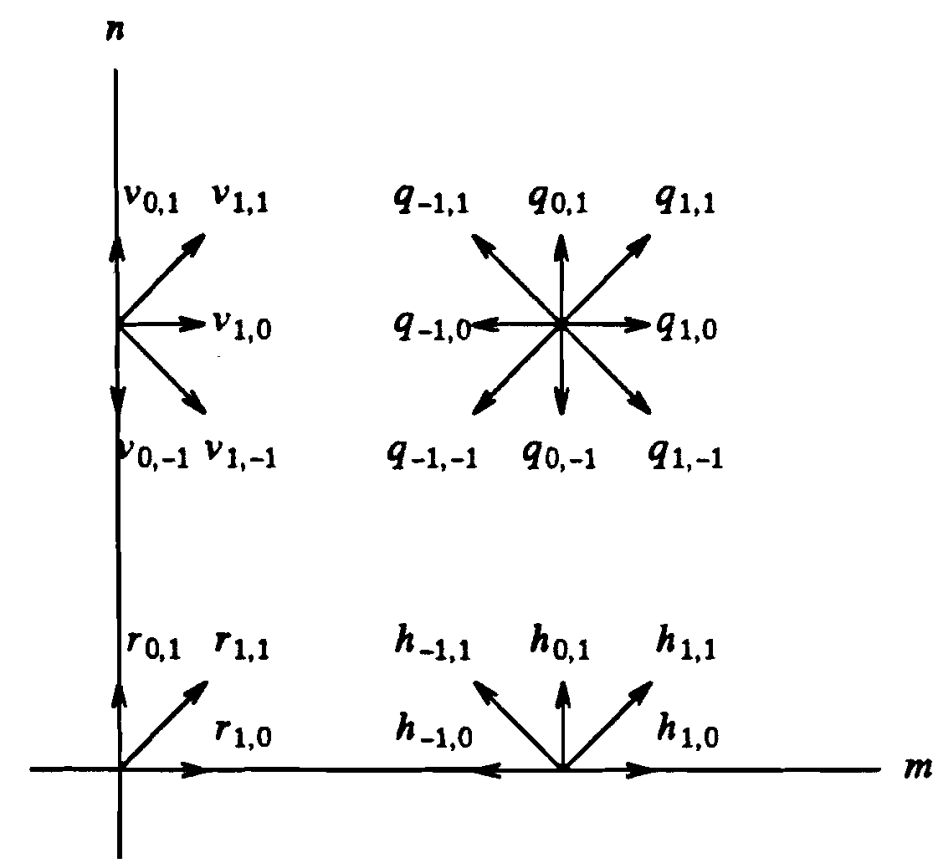

Figure 1: The transition rate diagram for a Markov process with constant rates and transitions restricted to neighboring states only. $q_{k, l}$ is the transition rate from $(m, n)$ to $(m+k, n+l)$ with $m, n>0$. A similar notation is used for transitions starting in boundary points.

\section{The model and the equilibrium equations}

The main purpose of the paper is to explore the basic conditions for random walks on twodimensional grids to have equilibrium distributions which can be represented as infinite series of product forms. Therefore the model is kept as simple as possible.

We consider continuous time Markov processes on the pairs $(m, n)$ of nonnegative integers which have the property that transitions can only take place to adjoining points (horizontally, vertically, and diagonally) in such a way that the rates for transitions starting at inner points do not depend on the starting point and similarly for both boundaries. The transition possibilities and rates are depicted in Figure 1. In fact, our Markov processes are continuous time random walks on a grid.

The rationale for this choice is the following. The symmetric shortest queue problem as well as Hofri's multiprogramming problem satisfy the conditions of this model, although the shortest queue problem needs a transformation first (see [2]). For the shortest queue problem, $m$ denotes the queue-length of the shortest queue, whereas $n$ denotes the excesslength of the second queue (length second queue minus length first queue). We then have the grid $m \geq 0, n$ free. The points with $n<0$ can be eliminated exploiting the symmetry. For the asymmetric shortest queue problem this last step is not possible, so in that case we keep a Markov process on the grid $m \geq 0, n$ free, which behaves like a different random walk on each of the parts $n>0$ and $n<0$ with the line $n=0$ as a kind of frontier in between. In [4] it has been proven that the solution on each of the two parts can be written as an infinite linear combination of product forms. This makes it clear that for more complex situations than the ones treated in this paper a compensation approach will work. Also the restriction to transitions to neighboring points does not seem to be essential, however, it facilitates the handling of boundaries in a similar way as the restriction to processes on one quadrant only. 
We suppose that the equilibrium probabilities $\left\{p_{m, n}\right\}$ exist for this Markov process, which we suppose to be irreducible from now on. The equilibrium probabilities satisfy the following relations, which are obtained by equating for each state the average number of times per time unit the state is reached and the average number of times per time unit the state is left. The equilibrium equations for the states $(0,0),(1,0),(0,1)$ and $(1,1)$ are left out because of their minor importance to the analysis.

$$
\begin{aligned}
p_{m, n} q= & p_{m+1, n-1} q_{-1,1}+p_{m, n-1} q_{0,1}+p_{m-1, n-1} q_{1,1}+p_{m-1, n} q_{0,1}+ \\
& p_{m-1, n+1} q_{1,-1}+p_{m, n+1} q_{0,-1}+p_{m+1, n+1} q_{-1,-1}+p_{m+1, n} q_{-1,0}, \\
& m>1, n>1 \\
p_{1, n} q= & p_{2, n-1} q_{-1,1}+p_{1, n-1} q_{0,1}+p_{0, n-1} \nu_{1,1}+p_{0, n} \nu_{1,0}+p_{0, n+1} \nu_{1,-1}+ \\
& p_{1, n+1} q_{0,-1}+p_{2, n+1} q_{-1,-1}+p_{2, n} q_{-1,0}, \\
& n>1 \\
p_{0, n} \nu= & p_{1, n-1} q_{-1,1}+p_{0, n-1} \nu_{0,1}+p_{0, n+1} \nu_{0,-1}+p_{1, n+1} q_{-1,-1}+p_{1, n} q_{-1,0}, \\
& n>1 \\
p_{m, 1} p= & p_{m+1,0} h_{-1,1}+p_{m, 0} h_{0,1}+p_{m-1,0} h_{1,1}+p_{m-1,1} q_{1,0}+p_{m-1,2} q_{1,-1}+ \\
& p_{m, 2} q_{0,-1}+p_{m+1,2} q_{-1,-1}+p_{m+1,1} q_{-1,0}, \\
& m>1 \\
& p_{m-1,0} h_{1,0}+p_{m-1,1} q_{1,-1}+p_{m, 1} q_{0,-1}+p_{m+1,1} q_{-1,-1}+p_{m+1,0} h_{-1,0}, \\
& m>1
\end{aligned}
$$

where

$$
\begin{aligned}
& q=q_{-1,1}+q_{0,1}+q_{1,1}+q_{1,0}+q_{1,-1}+q_{0,-1}+q_{-1,-1}+q_{-1,0}, \\
& \nu=\nu_{0,1}+\nu_{1,1}+\nu_{1,0}+\nu_{1,-1}+\nu_{0,-1}, \\
& h=h_{-1,1}+h_{0,1}+h_{1,1}+h_{1,0}+h_{-1,0} .
\end{aligned}
$$

\section{The compensation approach}

In this section we will attempt to construct a formal solution to the set of equations (2.1)-(2.5) by combining products of the form $\alpha^{m} \beta^{n}$, which all fit equation (2.1) for the interior points of the grid. Inserting the product $\alpha^{m} \beta^{n}$ into equation (2.1) and then dividing both sides of that equation by the common factor $\alpha^{m-1} \beta^{n-1}$ leads to the following characterization:

Lemma 3.1 The product $\alpha^{m} \beta^{n}$ is a solution of equation (2.1) if and only if $\alpha$ and $\beta$ satisfy

$$
\begin{aligned}
\alpha \beta q= & \alpha^{2} q_{-1,1}+\alpha q_{0,1}+q_{1,1}+\beta q_{1,0}+\beta^{2} q_{1,-1}+\alpha \beta^{2} q_{0,-1}+ \\
& \alpha^{2} \beta^{2} q_{-1,-1}+\alpha^{2} \beta q_{-1,0} .
\end{aligned}
$$

Any linear combination of productforms $\alpha^{m} \beta^{n}$ with $\alpha$ and $\beta$ satisfying equation (3.1) is a solution of equation (2.1). The next step is to construct such linear combinations which also satisfy $(2.2)-(2.5)$. 
Let us start by considering an arbitrary productform $\alpha^{m} \beta^{n}$ with (possibly complex) $\alpha, \beta$ satisfying equation (3.1). Most likely, this form will not satisfy the vertical boundary equations (2.2) and (2.3). Therefore, the straightforward compensation idea would imply the adding of a compensating term $c \tilde{\alpha}^{m} \tilde{\beta}^{n}$ such that

$$
\alpha^{m} \beta^{n}+c \tilde{\alpha}^{m} \tilde{\beta}^{n}
$$

satisfies (2.1)-(2.3). However, it will appear that this choice does not provide sufficient freedom for an appropriate choice of $c, \tilde{\alpha}, \tilde{\beta}$. In fact, as we will see, $\tilde{\beta}$ is forced to be equal to $\beta$ and $\tilde{\alpha}$ should constitute together with $\alpha$ the roots of (3.1) for the given $\beta$, only leaving $c$ for fulfilling two requirements. Therefore, we immediately choose a compensation procedure which is slightly more complicated:

Find $c, \tilde{\alpha}, \tilde{\beta}, e, \hat{\beta}$, such that

$$
z_{m, n}= \begin{cases}\alpha^{m} \beta^{n}+c \tilde{\alpha}^{m} \tilde{\beta}^{n} & \text { for } m>0, n>0 \\ e \hat{\beta}^{n} & \text { for } m=0, n>0\end{cases}
$$

satisfies (2.1)-(2.3).

A more elegant and more structural approach would be to introduce an extra dimension in the state space. The states would then become $(m, n, l)$ with $l \in\{0,1\}, l$ being 0 when $n=0$ and $l$ being 1 otherwise. Subsequently we would introduce productform solutions $\alpha^{m} \beta^{n} \gamma^{l}$ which satisfy (2.1) for $l=1$ and compensated solutions

$$
\alpha^{m} \beta^{n} \gamma^{l}+c \tilde{\alpha}^{m} \tilde{\beta}^{n} \tilde{\gamma}^{l}
$$

which should satisfy (2.1)-(2.3) with appropriately chosen values for $l$. However, for this first exploration, we prefer the more down-to-earth approach as specified earlier.

Insertion of the formulae for $z_{m, n}$ into equation (2.2) gives a condition of the following form:

$$
\beta^{n-1} A+c \tilde{\beta}^{n-1} B=e \hat{\beta}^{n-1} C \quad \text { for } n>1 .
$$

Except when coefficients are equal to zero, this condition requires

$$
\beta=\tilde{\beta}=\hat{\beta} .
$$

After exploiting (3.1), we find for $A$ :

$$
A=q_{1,1}+\beta q_{1,0}+\beta^{2} q_{1,-1} .
$$

For $B$ the same formula is obtained with $\beta$ replaced by $\tilde{\beta}$ (note that also $\tilde{\alpha}$ and $\tilde{\beta}$ satisfy (3.1)). For $C$ the $q$ should be replaced by $\nu$ and $\beta$ by $\hat{\beta}$.

$A$ is only equal to zero for two specific values of $\beta$ or it is identical to zero. In order to avoid all sorts of special cases, at this moment we suppose for the time being that $A, B, C$ and similar terms are not identical to zero:

\section{Assumption}

(i) $q_{1,1}+q_{1,0}+q_{1,-1}>0$ (there is a rate component to the east);

(ii) $q_{-1,1}+q_{-1,0}+q_{-1,-1}>0$

(there is a rate component to the west); 
(iii) $q_{-1,1}+q_{0,1}+q_{1,1}>0$

(there is a rate component to the north);

(iv) $q_{-1,-1}+q_{0,-1}+q_{1,-1}>0$

(there is a rate component to the south);

(v) $\nu_{1,1}+\nu_{1,0}+\nu_{1,-1}>0$

(reflecting $n$-axis);

(vi) $h_{-1,1}+h_{0,1}+h_{1,1}>0$

(reflecting m-axis).

Under this assumption, it may still happen that $A, B$ or $C$ is incidentally equal to zero, but we will first proceed in a formal way without taking that possibility into account and check this point later. Hence, we conclude that $\tilde{\beta}$ and $\hat{\beta}$ should be equal to $\beta$. Since $\tilde{\alpha}$ and $\tilde{\beta}$ have to satisfy (3.1), it follows that $\tilde{\alpha}$ must be the companion solution to $\alpha$ of (3.1) given $\beta$.

Insertion of the formulae for $z_{m, n}$ into equation (2.3) gives a similar equation as (3.2). Altogether this gives two linear equations in $C$ and $e$ which can easily be solved.

For the horizontal boundary equations (2.4) and (2.5) a similar approach could have been chosen which would have led to adding $d \alpha^{m} \beta^{n}$ instead of $c \tilde{\alpha}^{m} \beta^{n}$ to the original solution $\alpha^{m} \beta^{n}$ of equation (2.1), where $\underline{\beta}$ and $\beta$ are the solutions of the quadratic equation (3.1) for given $\alpha$.

This procedure is summarized in the following lemma:

\section{Lemma 3.2}

(i) Let $x_{1}$ and $x_{2}$ be the roots of the quadratic equation (9.1) for fixed $\beta$ and let

$$
z_{m, n}= \begin{cases}x_{1}^{m} \beta^{n}+c x_{2}^{m} \beta^{n}, & m>0, n>0, \\ e \beta^{n}, & m=0, n>0 .\end{cases}
$$

Then $z_{m, n}$ satisfies (2.1), (2.2) and (2.3), if $c$ and $e$ are chosen as follows:

$$
\begin{aligned}
& c=-\frac{\frac{\beta^{2} \nu_{1,-1}+\beta \nu_{1,0}+\nu_{1,1}}{x_{2}}+\nu_{0,1}+\beta^{2} \nu_{0,-1}-\beta \nu}{\frac{\beta^{2} \nu_{1,-1}+\beta \nu_{1,0}+\nu_{1,1}}{x_{1}}+\nu_{0,1}+\beta^{2} \nu_{0,-1}-\beta \nu}, \\
& e=-\frac{\left(\beta^{2} q_{1,-1}+\beta q_{1,0}+q_{1,1}\right)\left(\frac{1}{x_{2}}-\frac{1}{x_{1}}\right)}{\frac{\beta^{2} \nu_{1,-1}+\beta \nu_{1,0}+\nu_{1,1}}{x_{1}}+\nu_{0,1}+\beta^{2} \nu_{0,-1}-\beta \nu} .
\end{aligned}
$$

(ii) Let $y_{1}$ and $y_{2}$ be the roots of the quadratic equation (9.1) for fixed $\alpha$ and let

$$
w_{m, n}= \begin{cases}\alpha^{m} y_{1}^{n}+d \alpha^{m} y_{2}^{n}, & m>0, n>0, \\ f \alpha^{m}, & m>0, n=0 .\end{cases}
$$

Then $w_{m, n}$ satisfies (2.1), (2.4) and (2.5), if $d$ and $f$ are chosen as follows:

$$
d=-\frac{\frac{\alpha^{2} h_{-1,1}+\alpha h_{0,1}+h_{1,1}}{y_{2}}+h_{1,0}+\alpha^{2} h_{-1,0}-\alpha h}{\frac{\alpha^{2} h_{-1,1}+\alpha h_{0,1}+h_{1,1}}{y_{1}}+h_{1,0}+\alpha^{2} h_{-1,0}-\alpha h},
$$




$$
f=-\frac{\left(\alpha^{2} q_{-1,1}+\alpha q_{0,1}+q_{1,1}\right)\left(\frac{1}{y_{2}}-\frac{1}{y_{1}}\right)}{\frac{\alpha^{2} h_{-1,1}+\alpha h_{0,1}+h_{1,1}}{y_{1}}+h_{1,0}+\alpha^{2} h_{-1,0}-\alpha h} .
$$

Lemma 3.2 shows that an arbitrary solution of equation (2.1) can be compensated in such a way that it either becomes a solution to $(2.1),(2.2),(2.3)$ (Lemma 3.2(i)) or a solution to (2.1), (2.4), (2.5) (Lemma 3.2(ii)). One might hope that alternative use of these two compensation procedures would in the long run turn some solution to equation (2.1) into a solution of (2.1)-(2.5), at least if the compensating terms converge to zero sufficiently fast. For the moment being, we do not attend to the convergence problem, but only define the formal solution which can be constructed with this compensation approach.

Starting with an arbitrary product form solution $\alpha_{0}^{m} \beta_{0}^{n}$ of equation (2.1), we add $c_{1} \alpha_{1}^{m} \beta_{0}^{n}$ to compensate for the error of $\alpha_{0}^{m} \beta_{0}^{n}$ on the vertical boundary and, by doing so, we introduce a new error on the horizontal boundary, since $c_{1} \alpha_{1}^{m} \beta_{0}^{n}$ violates these boundary conditions. To compensate for this error we add $c_{1} d_{1} \alpha_{1}^{m} \beta_{1}^{n}$ where $\beta_{1}$ is the other root of (3.1) with $\alpha=\alpha_{1}$. The coefficient $d_{1}$ follows from Lemma 3.2(ii). However, this term violates the vertical boundary conditions, so we have to add again a term, and so on. Thus the compensation of $\alpha_{0}^{m} \beta_{0}^{n}$ on the vertical boundary generates an infinite sequence of compensation terms. An analogous sequence is generated by starting the compensation of $\alpha_{0}^{m} \beta_{0}^{n}$ on the horizontal boundary. This results in the following sum of terms:

$$
\cdots+\underbrace{d_{-1} c_{-1} \alpha_{-1}^{m} \beta_{-1}^{n}+\overbrace{d_{-1} c_{0} \alpha_{0}^{m} \beta_{-1}^{n}}^{H}+\underbrace{d_{0} c_{0} \alpha_{0}^{m} \beta_{0}^{n}}_{V}+\overbrace{d_{0} c_{1} \alpha_{1}^{m} \beta_{0}^{n}}^{H}+d_{1} c_{1} \alpha_{1}^{m} \beta_{1}^{n}}_{V}+\cdots
$$

By definition we have $c_{0}=d_{0}=1$. Each term in the sum satisfies (2.1), each sum of two terms with the same $\beta$-factor satisfies the vertical boundary conditions $(2.2)-(2.3)$ and each sum of two terms with the same $\alpha$-factor satisfies the horizontal boundary conditions (2.4)-(2.5). Since the equilibrium equations are linear, we can conclude that the infinite sum formally satisfies the equations $(2.1)-(2.5)$. Let us define $x_{m, n}\left(\alpha_{0}, \beta_{0}\right)$ as the infinite sum of compensation terms. For all $m>0, n>0$ set

$$
\begin{aligned}
x_{m, n}\left(\alpha_{0}, \beta_{0}\right) & =\sum_{i=-\infty}^{\infty} d_{i}\left(c_{i} \alpha_{i}^{m}+c_{i+1} \alpha_{i+1}^{m}\right) \beta_{i}^{m} \quad \text { (pairs with same } \beta \text {-factor) } \\
& =\sum_{i=-\infty}^{\infty} c_{i+1}\left(d_{i} \beta_{i}^{n}+d_{i+1} \beta_{i+1}^{n}\right) \alpha_{i+1}^{m} \quad \text { (pairs with same } \alpha \text {-factor) } .
\end{aligned}
$$

The pairs in (3.7) and (3.8) reflect the compensation on the vertical and horizontal boundaries, respectively. The compensation on these boundaries requires the introduction of new coefficients for the terms in $x_{0, n}\left(\alpha_{0}, \beta_{0}\right)$ and $x_{m, 0}\left(\alpha_{0}, \beta_{0}\right)$. For all $m=0, n>0$ set

$$
x_{0, n}\left(\alpha_{0}, \beta_{0}\right)=\sum_{i=-\infty}^{\infty} d_{i} e_{i} \beta_{i}^{n}
$$

and for all $m>0, n=0$

$$
x_{m, 0}\left(\alpha_{0}, \beta_{0}\right)=\sum_{i=-\infty}^{\infty} c_{i+1} f_{i+1} \alpha_{i+1}^{m} .
$$


Note that we did not introduce $x_{0,0}$. The main reason is that the equilibrium equations in and around the origin have been deleted.

Below we will formulate the recursion relations for $\alpha_{i}, \beta_{i}, c_{i}, d_{i}, e_{i}$, and $f_{i}$.

For the initial roots $\alpha_{0}$ and $\beta_{0}$ of the quadratic equation (3.1), the sequence

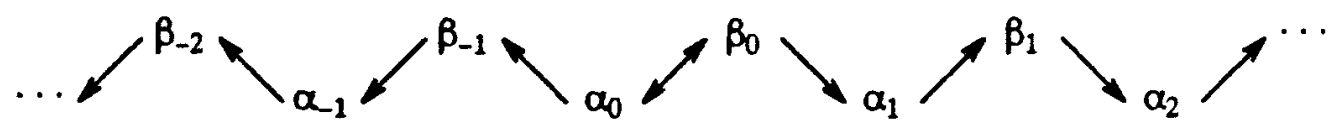

is generated such that for all $i$ the numbers $\alpha_{i}$ and $\alpha_{i+1}$ are the roots of (3.1) with fixed $\beta=\beta_{i}$, and $\beta_{i}$ and $\beta_{i+1}$ are the roots of (3.1) with fixed $\alpha=\alpha_{i+1}$.

The coefficients $c_{i}$ and $e_{i}$ are generated such that $\left(c_{i} \alpha_{i}^{m}+c_{i+1} \alpha_{i+1}^{m}\right) \beta_{i}^{n}$ and $e_{i} \beta_{i}^{n}$ satisfy the vertical boundary conditions (2.2)-(2.3). Initially set

$$
c_{0}=1 \text {. }
$$

Applying Lemma 3.2(i), the coefficients $c_{i+1}$ and $e_{i}$ for $i \geq 0$ can be obtained from $c_{i}$ by

$$
\begin{gathered}
c_{i+1}=-\frac{\frac{\beta_{i}^{2} \nu_{1,-1}+\beta_{i} \nu_{1,0}+\nu_{1,1}}{\alpha_{i+1}}+\nu_{0,1}+\beta_{i}^{2} \nu_{0,-1}-\beta_{i} \nu}{\frac{\beta_{i}^{2} \nu_{1,-1}+\beta_{i} \nu_{1,0}+\nu_{1,1}}{\alpha_{i}}+\nu_{0,1}+\beta_{i}^{2} \nu_{0,-1}-\beta_{i} \nu} c_{i}, i \geq 0, \\
e_{i}=-\frac{\left(\beta_{i}^{2} q_{1,-1}+\beta_{i} q_{1,0}+q_{1,1}\right)\left(\frac{1}{\alpha_{i+1}}-\frac{1}{\alpha_{i}}\right)}{\frac{\beta_{i}^{2} \nu_{1,-1}+\beta_{i} \nu_{1,0}+\nu_{1,1}}{\alpha_{i}}+\nu_{0,1}+\beta_{i}^{2} \nu_{0,-1}-\beta_{i} \nu} c_{i}, i \geq 0,
\end{gathered}
$$

and analogously $c_{i}$ and $e_{i}$ for $i<0$ can be obtained from $c_{i+1}$ by

$$
\begin{gathered}
c_{i}=-\frac{\frac{\beta_{i}^{2} \nu_{1,-1}+\beta_{i} \nu_{1,0}+\nu_{1,1}}{\alpha_{i}}+\nu_{0,1}+\beta_{i}^{2} \nu_{0,-1}-\beta_{i} \nu}{\frac{\beta_{i}^{2} \nu_{1,-1}+\beta_{i} \nu_{1,0}+\nu_{1,1}}{\alpha_{i+1}}+\nu_{0,1}+\beta_{i}^{2} \nu_{0,-1}-\beta_{i} \nu} c_{i+1}, i<0, \\
e_{i}=-\frac{\left(\beta_{i}^{2} q_{1,-1}+\beta_{i} q_{1,0}+q_{1,1}\right)\left(\frac{1}{\alpha_{i}}-\frac{1}{\alpha_{i+1}}\right)}{\frac{\beta_{i}^{2} \nu_{1,-1}+\beta_{i} \nu_{1,0}+\nu_{1,1}}{\alpha_{i+1}}+\nu_{0,1}+\beta_{i}^{2} \nu_{0,-1}-\beta_{i} \nu} c_{i+1}, i<0 .
\end{gathered}
$$

The coefficients $d_{i}$ and $f_{i}$ are generated such that $\left(d_{i} \beta_{i}^{n}+d_{i+1} \beta_{i+1}^{n}\right) \alpha_{i+1}^{m}$ and $f_{i+1} \alpha_{i+1}^{m}$ satisfy the horizontal boundary conditions (2.4)-(2.5). Initially set

$$
d_{0}=1 \text {. }
$$

Applying Lemma 3.2(ii), the coefficients $d_{i+1}$ and $f_{i+1}$ for $i \geq 0$ can be obtained from $d_{i}$ by

$$
\begin{gathered}
d_{i+1}=-\frac{\frac{\alpha_{i+1}^{2} h_{-1,1}+\alpha_{i+1} h_{0,1}+h_{1,1}}{\beta_{i+1}}+h_{1,0}+\alpha_{i+1}^{2} h_{-1,0}-\alpha_{i+1} h}{\frac{\alpha_{i+1}^{2} h_{-1,1}+\alpha_{i+1} h_{0,1}+h_{1,1}}{\beta_{i}}+h_{1,0}+\alpha_{i+1}^{2} h_{-1,0}-\alpha_{i+1} h} d_{i}, \quad i \geq 0, \\
f_{i+1}=-\frac{\left(\alpha_{i+1}^{2} q_{-1,1}+\alpha_{i+1} q_{0,1}+q_{1,1}\right)\left(\frac{1}{\beta_{i+1}}-\frac{1}{\beta_{i}}\right)}{\frac{\alpha_{i+1}^{2} h_{-1,1}+\alpha_{i+1} h_{0,1}+h_{1,1}}{\beta_{i}}+h_{1,0}+\alpha_{i+1}^{2} h_{-1,0}-\alpha_{i+1} h} d_{i}, \quad i \geq 0,
\end{gathered}
$$


and analogously $d_{i}$ and $f_{i+1}$ for $i<0$ can be obtained from $d_{i+1}$ by

$$
\begin{gathered}
d_{i}=-\frac{\frac{\alpha_{i+1}^{2} h_{-1,1}+\alpha_{i+1} h_{0,1}+h_{1,1}}{\beta_{i}}+h_{1,0}+\alpha_{i+1}^{2} h_{-1,0}-\alpha_{i+1} h}{\frac{\alpha_{i+1}^{2} h_{-1,1}+\alpha_{i+1} h_{0,1}+h_{1,1}}{\beta_{i+1}}+h_{1,0}+\alpha_{i+1}^{2} h_{-1,0}-\alpha_{i+1} h} d_{i+1}, i<0, \\
f_{i+1}=-\frac{\left(\alpha_{i+1}^{2} q_{-1,1}+\alpha_{i+1} q_{0,1}+q_{1,1}\right)\left(\frac{1}{\beta_{i}}-\frac{1}{\beta_{i+1}}\right)}{\frac{\alpha_{i+1}^{2} h_{-1,1}+\alpha_{i+1} h_{0,1}+h_{1,1}}{\beta_{i+1}}+h_{1,0}+\alpha_{i+1}^{2} h_{-1,0}-\alpha_{i+1} h} d_{i+1}, i<0 .
\end{gathered}
$$

This concludes the definition of $x_{m, n}\left(\alpha_{0}, \beta_{0}\right)$. For any pair of roots $\alpha_{0}, \beta_{0}$ of equation (3.1) the series $x_{m, n}\left(\alpha_{0}, \beta_{0}\right)$ formally satisfies the equations (2.1)-(2.5). In the next section it will be investigated for what $\alpha_{0}, \beta_{0}$ the series $x_{m, n}\left(\alpha_{0}, \beta_{0}\right)$ converges.

Remark If the rates on the vertical boundary are the truncation of the rates in the interior points, that is, $\nu_{1 j}=q_{1 j}$, then $e_{i}=c_{i}+c_{i+1}$ for all $i$ and thus the series (3.9) is identical to (3.7) with $m=0$. An analogous remark holds if $h_{j 1}=q_{j 1}$.

\section{Convergence results}

Under favorable conditions $x_{m, n}\left(\alpha_{0}, \beta_{0}\right)$ reduces to a finite sum. This happens if $c_{i}$ or $d_{i}$ vanishes for some $i \geq 0$ and for some $i \leq 0$, which means that from there on no compensation is needed anymore (all succeeding coefficients vanish due to the recursion). A simple example is that of two independent $M|M| 1$-queues, each with workload $\rho$, where for $\alpha_{0}=\rho$ and $\beta_{0}=\rho$ no compensation is needed at all, so $x_{m, n}(\rho, \rho)$ reduces to

$$
x_{m, n}(\rho, \rho)=\rho^{m} \rho^{n}
$$

Under unfortunate conditions compensation fails. This happens if for some value of $i$ the equation (3.1) with fixed $\beta=\beta_{1}$ or fixed $\alpha=\alpha_{i+1}$ reduces to a linear equation, so the necessary second root does not exist. If the second root is equal to the first one, then it can be verified that the compensation procedure constructs the null solution. Furthermore, compensation fails if for some value of $i$ the denominator in the definition of the coefficients vanishes (cf. (3.11)-(3.13)).

Let us suppose in this section that for the initial $\alpha_{0}$ and $\beta_{0}$ in at least one direction infinitely many compensation terms are needed and that compensation is always feasible. We investigate under what conditions the infinite linear combination $x_{m, n}\left(\alpha_{0}, \beta_{0}\right)$ converges. For convergence of $x_{m, n}\left(\alpha_{0}, \beta_{0}\right)$ for fixed $m$ and $n$ we will require that $\alpha_{i}$ and $\beta_{i}$ tend to zero as $i$ tends to plus or minus infinity. For convergence of the sum of $x_{m, n}\left(\alpha_{0}, \beta_{0}\right)$ over all values $m$ and $n$ (necessary for normalization) we will require that $\left|\alpha_{i}\right|<1,\left|\beta_{i}\right|<1$ for all $i$.

Below we investigate the implications of these convergence requirements for the transition structure in the interior of the state space. Numerical experience suggests that the behaviour of $\alpha_{i}$ and $\beta_{i}$ in case that $q_{1,1}+q_{0,1}+q_{1,0}>0$ is essentially different from the behaviour of $\alpha_{i}$ and $\beta_{i}$ in case that $q_{1,1}+q_{0,1}+q_{1,0}=0$. In the first type of case, examples do not show convergence for $\alpha_{i}$, but rather a wild oscillating behaviour. However, in the second type of case, experiments show very fast convergence of $\alpha_{i}$ both for $i \rightarrow \infty$ 
and $i \rightarrow-\infty$. Indeed, it is simple to prove that $q_{1,1}+q_{0,1}+q_{1,0}=0$ is necessary for convergence to zero of $\alpha_{i}$ and $\beta_{i}$. Therefore, consider that $\alpha_{i}$ and $\alpha_{i+1}$ are the roots of the quadratic equation (3.1) for $\beta=\beta_{i}$ and use this fact to compute $\alpha_{i} \alpha_{i+1}$ and $\alpha_{i}+\alpha_{i+1}$. It will immediately be clear that $q_{1,1}=q_{0,1}=0$ is necessary for convergence to zero of $\alpha_{i}$ and $\beta_{i}$. In the same way $\beta_{i}$ and $\beta_{i+1}$ are the roots of (3.1) for $\alpha=\alpha_{i+1}$ and computation of $\beta_{i}+\beta_{i+1}$ and $\beta_{i} \beta_{i+1}$ shows that $q_{1,1}=q_{1,0}=0$ is necessary for convergence to zero of $\alpha_{i}$ and $\beta_{i}$. Concluding, we have proved:

Lemma 4.1 A necessary condition for convergence to zero of $\alpha_{i}$ and $\beta_{i}$ is

$$
q_{1,1}=q_{1,0}=q_{0,1}=0 \text {. }
$$

This condition says that for convergence to zero of $\alpha_{i}$ and $\beta_{i}$ there may not be a northeast component in the transition rates for inner grid points. Luckily, the case of two independent $M|M|$ 1-queues did not need any compensation at all, since Lemma 4.1 shows that compensation would not work in such a case. If we change the boundary conditions of two independent $M|M| 1$-queues in such a way that an idle server may help a busy one, then compensation would be welcome, but it would not work according Lemma 4.1 and indeed the solutions are more complicated in these cases (cf. Fayolle, Iasnogorodski [13] and Konheim, Meilijson, Melkman [21]). We will now investigate whether this condition guarantees that the convergence requirements are satisfied for any pair of roots $\left(\alpha_{0}, \beta_{0}\right)$ of (3.1) satisfying $\left|\alpha_{0}\right|<1,\left|\beta_{0}\right|<1$. Therefore we suppose that the condition is satisfied and moreover, to avoid exceptional situations, that there is a rate component to the south-west:

\section{Assumption}

(i) $q_{1,1}+q_{1,0}+q_{0,1}=0$

(there is no rate component to the north-east)

(ii) $q_{-1,-1}+q_{-1,0}+q_{0,-1}>0$

(there is a rate component to the south-west)

Note that this assumption together with the assumption of Section 3 implies:

$$
q_{1,-1}>0, \quad q_{-1,1}>0
$$

Part (i) of the assumption leads to a considerable simplification of the double-quadratic equation (3.1) for $\alpha$ and $\beta$ which provides a solution to equilibrium equation (2.1) for inner grid points. This enables us to prove the following properties for the roots, where it is assumed that (3.1) has always two roots for $\alpha$ when $\beta$ is fixed (and the same of $\alpha$ and $\beta$ interchanged) by taking $\infty$ as the second root if the equation degenerates to a linear one.

Lemma 4.2 For each fixed $\alpha$ satisfying $0<|\alpha|<1$, equation (3.1) has exactly one root $\beta$ with $|\alpha|<|\beta|$ and one root $\beta$ with $|\alpha|>|\beta|>0$. The same holds with $\alpha$ and $\beta$ interchanged.

Instead of trying to prove this lemma directly from the solutions of the quadratic equations, it is easier to first rewrite equation (3.1) as an equation in $z=\frac{\beta}{\alpha}$ and then apply Rouché's theorem.

The result of Lemma 4.2 directly leads to some properties of the sequences $\left\{\alpha_{j}\right\},\left\{\beta_{j}\right\}$ generated by the compensation procedure as formulated in Section 3: 
Lemma 4.3 Let $\alpha_{0}$ and $\beta_{0}$ be nonzero roots of equation (9.1), satisfying $1>\left|\alpha_{0}\right|>$ $\left|\beta_{0}\right|>0$. Then there exists a negative value of $i$ for which $\left|\alpha_{i}\right| \geq 1$ or $\left|\beta_{i}\right| \geq 1$ and

$$
1>\left|\alpha_{i+1}\right|>\left|\beta_{i+1}\right|>\cdots>\left|\alpha_{0}\right|>\left|\beta_{0}\right|>\left|\alpha_{1}\right|>\left|\beta_{1}\right| \cdots \downarrow 0
$$

$A$ similar result holds if $\alpha_{0}$ and $\beta_{0}$ satisfy $1>\left|\beta_{0}\right|>\left|\alpha_{0}\right|>0$.

Proof The monotonicity directly follows from Lemma 4.2. To prove that there exists a negative value of $i$ for which $\left|\alpha_{i}\right| \geq 1$ or $\left|\beta_{i}\right| \geq 1$, we also need information about the $\beta$-roots of equation (3.1) for fixed $\alpha$ with $|\alpha|=1$.

For fixed $\alpha$ on the unit circle and $\alpha$ is not 1 or -1 it follows by applying Rouche's theorem, similarly as in the proof of Lemma 4.2, that equation (3.1) has one root $\beta$ with $|\beta|<|\alpha|$ and one root $\beta$ with $|\beta|>|\alpha|$. The same result holds for $\alpha=-1$ if at least one of the rates $q_{-1,0}$ or $q_{0,-1}$ is positive. For fixed $\alpha=1$ and also for $\alpha=-1$, if $q_{-1,0}=q_{0,-1}=0$, equation (3.1) is solved by $\beta=\alpha$ and $\beta=\alpha q_{-1,1} /\left(q_{-1,-1}+q_{0,-1}+q_{1,-1}\right)$. Hence, if $q_{-1,1}<q_{-1,-1}+q_{0,-1}+q_{1,-1}$, then we can call $\beta(\alpha)$ the root of (3.1) for fixed $\alpha$ with $|\alpha|<1$, which satisfies $|\beta(\alpha)|<|\alpha|$. $\beta(\alpha)$ is properly defined for $|\alpha| \leq 1$. $\beta(\alpha) / \alpha$ is continuous and hence the maximum of $|\beta(\alpha) / \alpha|$ exists and is less than one. So, $\left|\beta_{i} / \alpha_{i}\right|=\left|\beta\left(\alpha_{i}\right) / \alpha_{i}\right| \leq \max _{|\alpha| \leq 1}|\beta(\alpha) / \alpha|<1$ as long as $\left|\alpha_{i}\right|<1$. This proves that $\left|\alpha_{i}\right|$ and $\left|\beta_{i}\right|$ decrease exponentially fast to zero as $i$ tends to plus infinity, and that $\left|\alpha_{i}\right| \geq 1$ or $\left|\beta_{i}\right| \geq 1$ for some negative value of $i$.

If $q_{-1,1} \geq q_{-1,-1}+q_{0,-1}+q_{1,-1}$, then $q_{1,-1}<q_{-1,-1}+q_{-1,0}+q_{-1,1}$, which follows from the second part of the assumption. So we can repeat the above arguments by considering the roots of (3.1) for fixed $\beta$ instead of fixed $\alpha$.

Note that Lemma 4.3 covers all relevant cases, since the case $0<\left|\alpha_{0}\right|=\left|\beta_{0}\right|<1$ is excluded by Lemma 4.2. When the sequence of $\alpha_{i}$ and $\beta_{i}$ is started with nonzero roots $\alpha_{0}$ and $\beta_{0}$ each inside the unit circle, then, according to Lemma 4.3, $\left|\alpha_{i}\right|$ and $\left|\beta_{i}\right|$ monotonously decrease to zero in at least one direction. In the opposite direction $\left|\alpha_{i}\right|$ and $\left|\beta_{i}\right|$ are increasing as long as $\alpha_{i}$ and $\beta_{i}$ are in the open unit disk, but eventually, $\left|\alpha_{i}\right| \geq 1$ or $\left|\beta_{i}\right| \geq 1$. Therefore we cannot meet the convergence requirements in that direction, unless in that direction $c_{i}$ or $d_{i}$ vanishes for some $i$ before $\alpha_{i}$ or $\beta_{i}$ runs out of the open unit disk. After renumbering the terms this amounts to the requirement that the initial product $\alpha_{0}^{m} \beta_{0}^{n}$ fits the horizontal boundary conditions $\left(d_{-1}=0\right)$ if $\left|\alpha_{0}\right|>\left|\beta_{0}\right|$ or otherwise the vertical boundary conditions $\left(c_{1}=0\right)$. In such a case we have to generate compensation terms in the decreasing direction only. Pairs $\alpha_{0}, \beta_{0}$ which satisfy these requirements will be called feasible pairs. That means, feasible pairs are roots of (3.1) satisfying $0<\left|\alpha_{0}\right|<1$ and $0<\left|\beta_{0}\right|<1$, such that $\alpha_{0}^{m} \beta_{0}^{n}$ either satisfies the horizontal boundary conditions (if $\left|\alpha_{0}\right|>\left|\beta_{0}\right|$ ) or the vertical boundary conditions (if $\left|\beta_{0}\right|>\left|\alpha_{0}\right|$ ). In the first case we have $d_{-1}=0$ and in the second case $c_{i}=0$.

We end this section with a theorem stating that $\alpha_{0}, \beta_{0}, \alpha_{1}, \beta_{1}, \ldots$ can be solved explicitly if $1>\left|\alpha_{0}\right|>\left|\beta_{0}\right|$. The same result holds for $\beta_{0}, \alpha_{0}, \beta_{-1}, \alpha_{-1}$ if $1>\left|\beta_{0}\right|>\left|\alpha_{0}\right|$. The result generalises lemma 3 in Kingman [20] and also the proof is a direct extension of Kingman's proof. 
Theorem 4.1 (Explicit solution of $\alpha_{i}$ and $\beta_{i}$ ) Let $\alpha_{0}$ and $\beta_{0}$ be nonzero roots inside the unit circle of equation (8.1) and $\left|\alpha_{0}\right|>\left|\beta_{0}\right|$. Then there exist complex numbers $a$ and $b$, depending on $\alpha_{0}$ and $\beta_{0}$, such that for $i \geq 0$

$$
\begin{aligned}
& \frac{1}{\alpha_{i}}=A+\sqrt{\gamma}\left(a \lambda^{i}+\frac{1}{a \lambda^{i}}\right), \\
& \frac{1}{\beta_{i}}=B+\sqrt{\delta}\left(b \lambda^{i}+\frac{1}{b \lambda^{i}}\right),
\end{aligned}
$$

where

$$
\begin{aligned}
& A=\frac{q q_{-1,0}+2 q_{0,-1} q_{-1,1}}{q^{2}-4 q_{1,-1} q_{-1,1}}, \\
& B=\frac{q q_{0,-1}+2 q_{-1,0} q_{1,-1}}{q^{2}-4 q_{1,-1} q_{-1,1}}, \\
& \gamma=\frac{A^{2} q_{1,-1} q_{-1,1}}{q^{2}}+\frac{q_{-1,1} q_{-1,-1} q^{2}+q_{-1,1} q_{0,-1}\left(q q_{-1,0}+q_{-1,1} q_{0,-1}\right)}{q^{2}\left(q^{2}-4 q_{1,-1} q_{-1,1}\right)} \\
& \delta=\frac{B^{2} q_{1,-1} q_{-1,1}}{q^{2}}+\frac{q_{1,-1} q_{-1,-1} q^{2}+q_{1,-1} q_{-1,0}\left(q q_{0,-1}+q_{1,-1} q_{-1,0}\right)}{q^{2}\left(q^{2}-4 q_{1,-1} q_{-1,1}\right)} \\
& \lambda=\frac{q-\sqrt{q^{2}-4 q_{1,-1} q_{-1,1}}}{q+\sqrt{q^{2}-4 q_{1,-1} q_{-1,1}}} .
\end{aligned}
$$

Proof We prove the expressions for $1 / \alpha_{i}$. The expressions for $1 / \beta_{i}$ can be obtained similarly (replace $q_{i j}$ by $q_{j i}$ ). By Lemma 4.3 the numbers $\alpha_{i}$ and $\beta_{i}$ are nonzero for $i \geq 0$, and equation (3.1) does not reduce to a linear equation for fixed $\beta=\beta_{i}$ or $\alpha=\alpha_{i+1}$, so the denominator in the formulae for $\alpha_{i}+\alpha_{i+1}$ and $\alpha_{i} \alpha_{i+1}$, as used in the proof of Lemma 4.1 (similarly for $\beta_{i}+\beta_{i+1}$ and $\beta_{i} \beta_{i+1}$ ), does not vanish for $i \geq 0$. Then, we obtain for $i \geq 0$ that (recall that $q_{1,1}=q_{1,0}=0$ ),

$$
\frac{1}{\alpha_{i}}+\frac{1}{\alpha_{i+1}}=\frac{1}{\beta_{i}} \frac{q}{q_{1,-1}}-\frac{q_{0,-1}}{q_{1,-1}} .
$$

Adding this relation for $i$ and $i+1$ yields for $i \geq 0$,

$$
\frac{1}{\alpha_{i}}+\frac{2}{\alpha_{i+1}}+\frac{1}{\alpha_{i+2}}=\left(\frac{1}{\beta_{i}}+\frac{1}{\beta_{i+1}}\right) \frac{q}{q_{1,-1}}-2 \frac{q_{0,-1}}{q_{1,-1}} .
$$

Similarly, we obtain for $i \geq 0$ the analogy of (4.5) for $1 / \beta_{i}$,

$$
\frac{1}{\beta_{i}}+\frac{1}{\beta_{i+1}}=\frac{1}{\alpha_{i+1}} \frac{q}{q_{-1,1}}-\frac{q_{-1,0}}{q_{-1,1}} .
$$

Inserting that equality into (4.6) gives for $i \geq 0$ that

$$
\frac{1}{\alpha_{i}}+\frac{2}{\alpha_{i+1}}+\frac{1}{\alpha_{i+2}}=\left(\frac{1}{\alpha_{i+1}} \frac{q}{q_{-1,1}}-\frac{q_{-1,0}}{q_{-1,1}}\right) \frac{q}{q_{1,-1}}-2 \frac{q_{0,-1}}{q_{1,-1}} .
$$

This is a second order inhomogeneous recursion relation for $\frac{1}{\alpha_{i}}$, whose solution is given by

$$
\frac{1}{\alpha_{i}}=A+a_{1} \lambda^{i}+a_{2} \lambda^{-i},
$$


where $A$ and $\lambda$ are given by (4.2) and (4.4) (note that by the assumption the denominator $q^{2}-4 q_{1,-1} q_{-1,1}$ is positive), and $a_{1}$ and $a_{2}$ are complex constants, which follow from the initial values $1 / \alpha_{0}$ and $1 / \alpha_{1}$. To establish (4.1) it remains to prove that $a_{1}$ and $a_{2}$ satisfy

$$
a_{1} a_{2}=\gamma
$$

First note that the formula for $\alpha_{i} \alpha_{i+1}$ is equivalent with

$$
\frac{1}{\alpha_{i}} \frac{1}{\alpha_{i+1}}=\frac{q_{-1,1}}{q_{1,-1}} \frac{1}{\beta_{i}^{2}}+\frac{q_{-1,0}}{q_{1,-1}} \frac{1}{\beta_{i}}+\frac{q_{-1,-1}}{q_{1,-1}} .
$$

Eliminating $1 / \beta_{i}$ from (4.5) and (4.8), leads to

$$
\begin{gathered}
\left(q_{1,-1} q^{2}-2 q_{1,-1}^{2} q_{-1,1}\right) \frac{1}{\alpha_{i}} \frac{1}{\alpha_{i+1}}=q_{-1,-1} q^{2}+q_{0,-1}\left(q q_{-1,0}+q_{-1,1} q_{0,1}\right)+ \\
\left(q_{1,-1}\left(q q_{-1,0}+q_{-1,1} q_{0,-1}\right)+q_{0,-1} q_{-1,1} q_{1,-1}\right)\left(\frac{1}{\alpha_{i}}+\frac{1}{\alpha_{i+1}}\right)+ \\
q_{1,-1}^{2} q_{-1,1}\left(\frac{1}{\alpha_{i}^{2}}+\frac{1}{\alpha_{i+1}^{2}}\right) .
\end{gathered}
$$

Inserting the form (4.7) for $1 / \alpha_{0}$ and $1 / \alpha_{1}$, equation (4.9) reduces to the identity $a_{1} a_{2}=\gamma$.

\section{The quest for feasible pairs}

After having investigated, in Section 4, the convergence conditions, the question arises whether there really exist feasible pairs which generate a convergent series. And if so, how many feasible pairs do exist? In this section we will derive conditions for the existence of feasible pairs and show that the possible number of feasible pairs directly follows from the transition structure at the boundaries. We will also show that all feasible pairs are real. We will only treat feasibility with respect to the horizontal boundary, that is we only consider roots $\alpha_{0}, \beta_{0}$ of (3.1) with $1>\left|\alpha_{0}\right|>\left|\beta_{0}\right|>0$ satisfying $d_{-1}=0$. The feasibility with respect to the vertical boundary can be treated symmetrically.

Theorem 5.1 The number of pairs $\alpha_{0}, \beta_{0}$ which are feasible with respect to the horizontal boundary is at most two. Such pairs are always realvalued.

Depending on the boundary behaviour, the following details hold:

(i) If $h_{1,1}>0$, then there may indeed exist two pairs. If this is the case, then at least one $\alpha_{0}$ (and one $\beta_{0}$ ) must be positive.

(ii) If $h_{1,1}=0$ and $h_{0,1}+h_{1,0}>0$, then there can at most be one pair. If this is the case, then the roots are positive.

(iii) If $h_{1,1}=h_{0,1}=h_{1,0}=0$, then no pairs exist.

Proof The third case is the simplest one. In that case it can easily be derived from the definition of $d_{-1}$ that there exist no feasible pairs. Both other cases may be treated by introducing an irreducible Markov process on a slightly different grid, namely $\{(m, n) \mid$ $m+n>0, n \geq 0\} \cup\{(-1,0),(0,0)\}$ for the case $h_{1,1}>0$. The transition rates from states with $m+n>0$ are given by $h_{k, l}$ if $n=0$, and by $q_{k, l}$ if $n>0$. We assume that from 


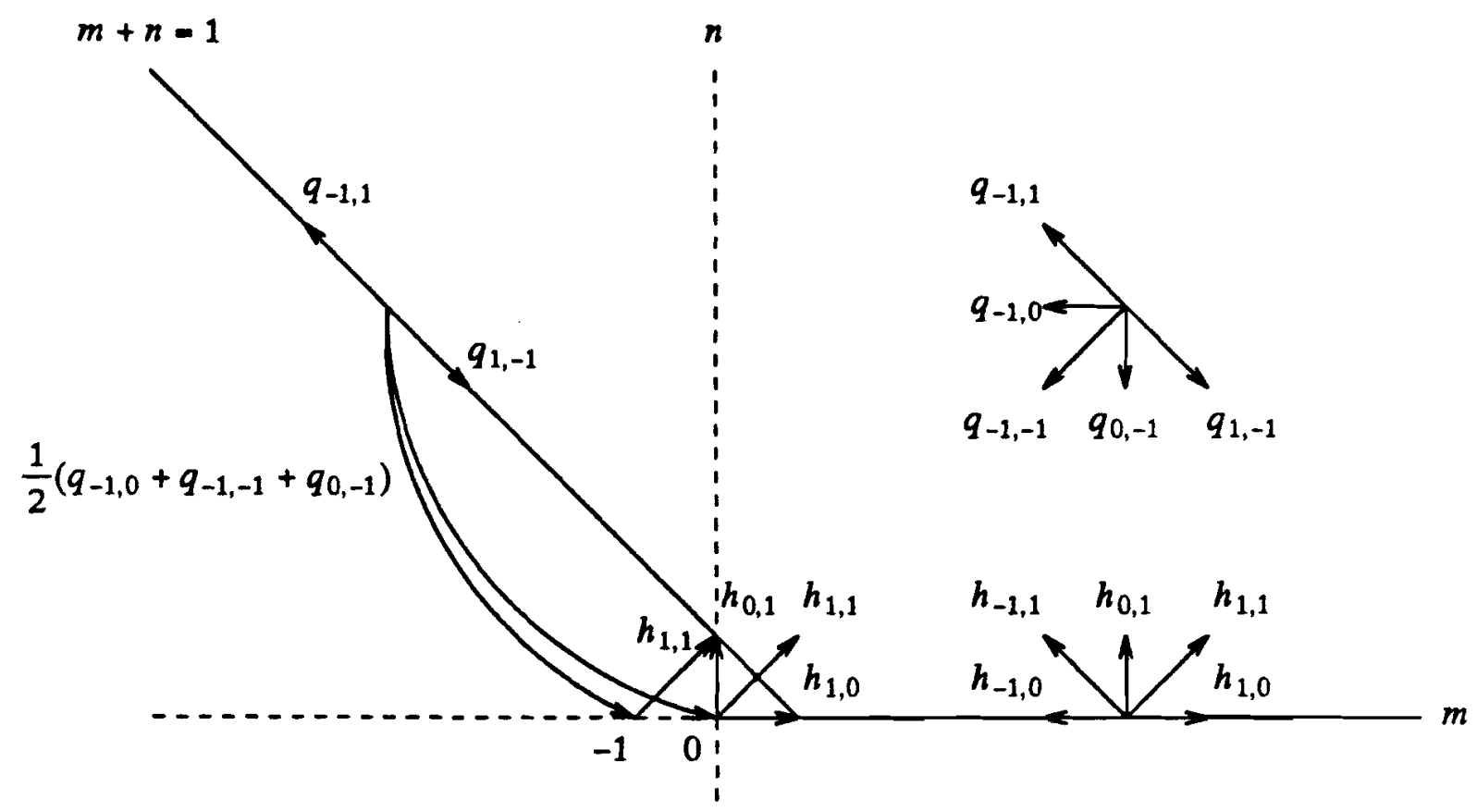

Figure 2: Transition rates for the Markov process in the proof of Theorem 5.1 on the set $\{(m, n), m+n \geq 0, n \geq 0\} \cup\{(-1,0),(0,0)\}$ for the case $h_{1,1}>0$.

states with $m+n=1$ and $n>0$ transitions are possible to $(-1,0)$ and $(0,0)$ with rate $\left(q_{-1,0}+q_{-1,-1}+q_{0,-1}\right) / 2$ to each of the two states. From state $(-1,0)$ and state $(0,0)$, the Markov process can reenter the set of states with $m+n>0$ with rate $h_{k, l}$ to $(0,1)$, and from state $(0,0)$ with rate $h_{1,1}$ to $(1,1)$. The transition rate diagram is depicted in Figure 2.

The equilibrium equation in state $(m, n)$ with $m+n>0$ is given by $(2.1)$ for $n>1$, (2.4) for $n=1$ and finally, by (2.5) for $n=0$. The equations in $(-1,0)$ and $(0,0)$ are different from (2.5), which is mainly due to the incoming rates from states with $m+n=1$. Hence, for each pair of roots $\alpha_{0}$ and $\beta_{0}$ of equation (3.1) with $1>\left|\alpha_{0}\right|>\left|\beta_{0}\right|>0$ satisfying $d_{-1}=0$, the product $z_{m, n}\left(\alpha_{0}, \beta_{0}\right)$, defined by

$$
z_{m, n}\left(\alpha_{0}, \beta_{0}\right)= \begin{cases}\alpha_{0}^{m} \beta_{0}^{n}, & m+n>0, n>0 \\ f_{0} \alpha_{0}^{m}, & m \geq-1, n=0\end{cases}
$$

satisfies the equilibrium equations in all states with $m+n>0$. Note that $z_{m, n}\left(\alpha_{0}, \beta_{0}\right)$ also satisfies the equilibrium equations on the boundary $m+n=1$, and therefore does not initiate the compensation method. Since $\left|\beta_{0}\right|<\left|\alpha_{0}\right|<1$, the product $z_{m, n}\left(\alpha_{0}, \beta_{0}\right)$ converges absolutely, that is

$$
\sum_{\substack{n+m>0 \\ n \geq 0}}\left|z_{m, n}\left(\alpha_{0}, \beta_{0}\right)\right|<\infty
$$

For each finite set of pairs $\alpha_{0}$ and $\beta_{0}$ the corresponding products $z_{m, n}\left(\alpha_{0}, \beta_{0}\right)$ are linearly independent on the set of states with $m+n>0$, which, by considering these products for fixed $m$ or $n$, follows from the fact that the linear equations for finding the weights in case of linear dependence would be characterised by the Vandermonde matrix, which is clearly nonsingular in the case of distinct entries.

The first question is how many feasible pairs there possibly exist. Suppose that $\left(\hat{\alpha}_{0}, \hat{\beta}_{0}\right)$ and $\left(\tilde{\alpha}_{0}, \tilde{\beta}_{0}\right)$ are feasible pairs. Then there exist nonnull coefficients $\hat{k}$ and $\tilde{k}$ such that 
the linear combination $\hat{k} z_{m, n}\left(\hat{\alpha}_{0}, \hat{\beta}_{0}\right)+\tilde{k} z_{m, n}\left(\tilde{\alpha}_{0}, \tilde{\beta}_{0}\right)$ fits the (homogeneous) equilibrium equation in state $(0,0)$. The remaining equation in state $(-1,0)$ is also satisfied, since inserting this linear combination of $z_{m, n}\left(\hat{\alpha}_{0}, \hat{\beta}_{0}\right)$ and $z_{m, n}\left(\tilde{\alpha}_{0}, \tilde{\beta}_{0}\right)$ into the equation on the set of states with $m+n \geq 0$ and $n \geq 0$ and then summing over these equations and changing summations, exactly yields the equilibrium equation in state $(-1,0)$. Changing summations is feasible by the absolute convergence in (5.1). So $\hat{k} z_{m, n}\left(\hat{\alpha}_{0}, \hat{\beta}_{0}\right)+\tilde{k} z_{m, n}\left(\tilde{\alpha}_{0}, \tilde{\beta}_{0}\right)$ is an absolutely convergent solution of the equilibrium equations of the Markov process in Figure 2 , and further, this linear combination is nonnull, since $z_{m, n}\left(\hat{\alpha}_{0}, \hat{\beta}_{0}\right)$ and $z_{m, n}\left(\tilde{\alpha}_{0}, \tilde{\beta}_{0}\right)$ are linearly independent. Hence, by a result of Foster ([16], Theorem 1), the Markov process in Figure 2 is ergodic and the linear combination $\hat{k} z_{m, n}\left(\hat{\alpha}_{0}, \hat{\beta}_{0}\right)+\hat{k} z_{m, n}\left(\tilde{\alpha}_{0}, \tilde{\beta}_{0}\right)$ can be normalized to produce the equilibrium distribution. Since the equilibrium distribution of an ergodic Markov process is unique and for different pairs of $\alpha_{0}$ and $\beta_{0}$ the products $z_{m, n}\left(\alpha_{0}, \beta_{0}\right)$ are linearly independent, we can conclude that there exist at most two feasible pairs.

The second question is whether there possibly exist complex feasible pairs of $\alpha_{0}$ and $\beta_{0}$. Suppose $\alpha_{0}$ is complex. Then there exists a nonnull coefficient $k$ such that the sum of $k z_{m, n}\left(\alpha_{0}, \beta_{0}\right)$ and the complex conjugate of this term satisfy all equations of the Markov process in Figure 2. However, for fixed $n \geq 0$ this sum is of the form

$$
K \alpha_{0}^{m}+\bar{K} \bar{\alpha}_{0}^{m}
$$

which has positive and negative values for $m>0$ and therefore cannot produce probabilities. Hence, $\alpha_{0}$ must be real. By a similar argument, it follows that $\beta_{0}$ must be real and moreover, if there exist two feasible pairs, then at least one of the $\alpha_{0}$ (and one of the $\beta_{0}$ ) must be positive.

This concludes the treatment of the case $h_{1,1}>0$. For the other open case a Markov process is introduced on the same grid minus the point $(-1,0)$. The rates which were split up between $(-1,0)$ and $(0,0)$ in Figure 2, are now completely directed towards $(0,0)$. Because of the analogy between both cases, the details will be omitted.

The next question, of course, is: do such feasible pairs really exist if $h_{0,1}+h_{1,1}+h_{1,0}>0$, that means in the first two cases of the preceeding theorem. The following theorem states that in the case $q_{-1,-1}+q_{0,-1}+q_{1,-1} \leq q_{-1,1}$ indeed the maximal numbers of feasible pairs are found and that in the other case the same is true if an extra condition is satisfied. Later on in this section it will appear that this extra condition is exactly Neuts' mean drift condition (see [20], Theorem 1.7.1).

Theorem 5.2 If $q_{-1,-1}+q_{0,-1}+q_{1,-1} \leq q_{-1,1}$, then the maximum numbers of feasible pairs with respect to the horizontal boundary are found (compare Theorem 5.1). If this maximum number is two, then one $\alpha_{0}$ is found in $(0,1)$ and one in $(-1,0)$. If this maximum number is only one, then this $\alpha_{0}$ lies in $(0,1)$.

If $q_{-1,-1}+q_{0,-1}+q_{1,-1}>q_{-1,1}$ and Condition $A$ (to be specified later) is satisfied, then also the maximum number of feasible pairs is found and they have the same properties as in the case mentioned first.

Proof The proof will not be given in full detail since it is rather lengthy but not very exciting. The proof essentially consists of a careful study of the behaviour of the $\beta_{1}$ roots of equation (3.1) as function $\alpha$ (and vice versa). We will highlight the proof as far as necessary to make it clear how and why condition A enters. Equation (3.13) gives the 
definition of $d_{i}$ for $i<0$ as a quotient. Therefore, for $d_{-1}$ to be zero it requires that the numerator of (3.13) is zero and the denominator is unequal to zero:

$$
\begin{aligned}
& \frac{\alpha_{0}^{2} h_{-1,1}+\alpha_{0} h_{0,1}+h_{1,1}}{\beta_{-1}}+h_{1,0}+\alpha_{0}^{2} h_{-1,0}-\alpha_{0} h=0, \\
& \frac{\alpha_{0}^{2} h_{-1,1}+\alpha_{0} h_{0,1}+h_{1,1}}{\beta_{0}}+h_{1,0}+\alpha_{0}^{2} h_{-1,0}-\alpha_{0} h \neq 0 .
\end{aligned}
$$

The roots $\beta_{0}$ and $\beta_{-1}$ will be regarded as functions of $\alpha_{0}$. By Theorem 5.1 the analysis can henceforth be restricted to real $\alpha_{0}$. It can readily be verified that inequality (5.3) is always satisfied for nonzero $\alpha_{0} \in(-1,1)$ which solve equation (5.2). To analyze (5.2) we insert the explicit formula for the root $\beta_{-1}$ of which we first mention some useful properties.

For fixed $\alpha$, equation (3.1) is solved by

$$
X_{ \pm}(\alpha)=\alpha \frac{q-\alpha q_{-1,0} \pm \sqrt{\left(q-\alpha q_{-1,0}\right)^{2}-4\left(\alpha^{2} q_{-1,-1}+\alpha q_{0,-1}+q_{1,-1}\right) q_{-1,1}}}{2\left(\alpha^{2} q_{-1,-1}+\alpha q_{0,-1}+q_{1,-1}\right)}
$$

The denominator in (5.4) possibly vanishes for some $\alpha<0$. There we can continuously extend $X_{-}(\alpha)$ and $\frac{1}{X_{+}(\alpha)}$ by taking $X_{-}(\alpha)=\frac{\alpha q-1,1}{\left(q-\alpha q_{-1,0}\right.}$ and $\frac{1}{X_{+}(\alpha)}=0$, thus $X_{+}(\alpha)=\infty$. For $X_{ \pm}$we state the following monotonicity properties without giving the proofs which are based on an analysis of the square root in equation (5.4):

Lemma 5.1 For all $0<\alpha<1$ the ratio $\frac{X_{+}(\alpha)}{\alpha}$ is decreasing and $\frac{X_{-}(\alpha)}{\alpha}$ increasing, and

$$
\left|X_{+}(-\alpha)\right| \geq X_{+}(\alpha)>\alpha>X_{-}(\alpha) \geq-X_{-}(-\alpha)>0
$$

These properties (and the symmetric ones for $\alpha$ given $\beta$ ) help us to refine Lemma 4.3 to:

Lemma 5.2 Let $\alpha_{0}$ and $\beta_{0}$ be roots of equation (3.1) satisfying $1>\left|\alpha_{0}\right|>\left|\beta_{0}\right|>0$. If $0<\alpha_{0}<1$, then

$$
\alpha_{0}>X_{-}\left(\alpha_{0}\right)=\beta_{0}>Y_{-}\left(\beta_{0}\right)=\alpha_{1}>X_{-}\left(\alpha_{1}\right)=\beta_{1}>\cdots>0 ;
$$

and if $-1<\alpha_{0}<0$, then

$$
\alpha_{0}<X_{-}\left(\alpha_{0}\right)=\beta_{0}<Y_{-}\left(\beta_{0}\right)=\alpha_{1}<X_{-}\left(\alpha_{1}\right)=\beta_{1}<\cdots<0 .
$$

Here $Y_{ \pm}(\beta)$ are the solutions of (\$.1) given $\beta$.

Since $\left|\alpha_{0}\right|>\left|\beta_{0}\right|$, it follows that $\left|\alpha_{0}\right|<\left|\beta_{-1}\right|$, so by Lemma (5.1) we can set $\beta_{-1}=X_{+}\left(\alpha_{0}\right)$ and $\beta_{0}=X_{-}\left(\alpha_{0}\right)$. Substituting $X_{+}\left(\alpha_{0}\right)$ for $\beta_{-1}$ in equation (5.2) and rearranging terms, we get that $\alpha_{0}$ has to be a root of the equation

$$
\alpha h=\frac{\alpha^{2} h_{-1,1}+\alpha h_{0,1}+h_{1,1}}{X_{+}(\alpha)}+\alpha^{2} h_{-1,0}+h_{1,0}
$$

Denote by $L H(\alpha)$ the left-hand side of (5.5) and by $R H(\alpha)$ the right-hand side. In Figure 3 we depict $L H(\alpha)$ and $R H(\alpha)$ for the case that $q_{-1,1}=q_{0,-1}=h_{-1,0}=2$, $q_{1,-1}=h_{1,1}=1$ and all other $q_{k l}$ and $h_{k l}$ are zero.

Figure 3 suggests that: 


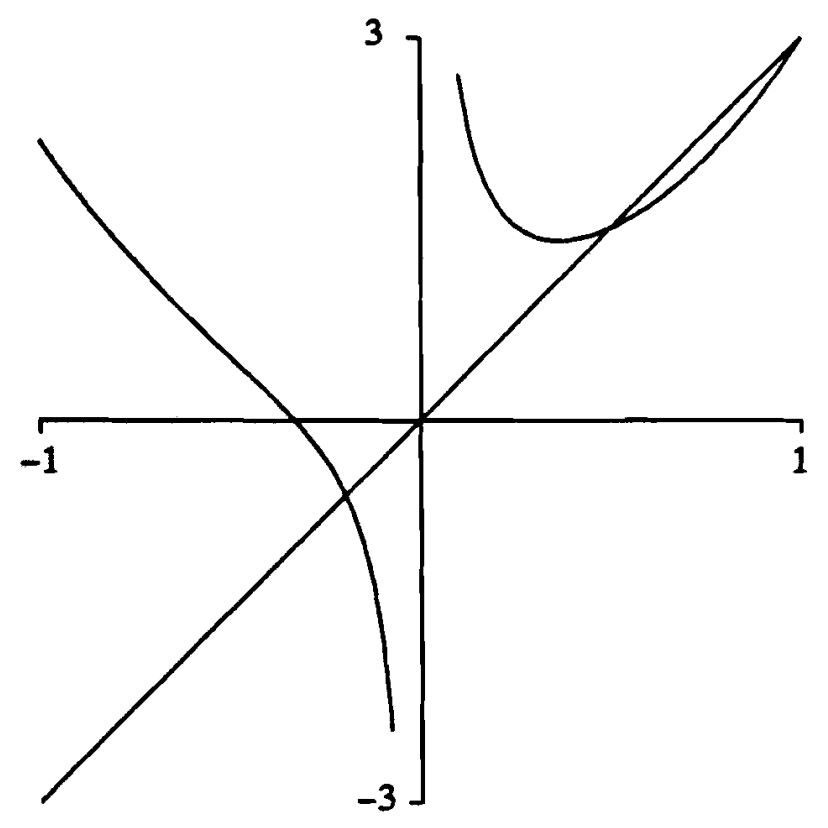

Figure 3: The left-hand side $L H(\alpha)$ and the right-hand side $R H(\alpha)$ of equation (5.5) for the case that $q_{-1,1}=q_{0,-1}=h_{-1,0}=2, q_{1,-1}=h_{1,1}=1$ and all other $q_{k l}$ and $h_{k l}$ are zero.

Lemma 5.3 The right-hand side $R H(\alpha)$ of equation (5.5) is strictly convex for $0<\alpha<1$.

The lemma easily follows once it has been shown that the square root in (5.4) is strictly concave for $0<\alpha<1$. The latter property can be derived by straightforward calculations.

It is easy to verify that at the boundaries $\alpha=0$ and $\alpha=1$,

$$
\begin{aligned}
R H\left(0^{+}\right) & >L H(0) & & \text { if } h_{1,1}+h_{0,1}+h_{1,0}>0 \\
& =L H(0) & & \text { otherwise } \\
R H(1) & <L H(1) & & \text { if } q_{-1,-1}+q_{0,-1}+q_{1,-1}>q_{-1,1} \\
& =L H(1) & & \text { otherwise. }
\end{aligned}
$$

Hence, in case $h_{1,1}+h_{0,1}+h_{1,0}>0$, there is a root of equation (5.5) in the interval $(0,1)$ if $q_{-1,-1}+q_{0,-1}+q_{1,-1}>q_{-1,1}$ and otherwise the following extra condition is required:

\section{Condition A}

$$
R H^{\prime}(1)>L H^{\prime}(1) \text {. }
$$

Remark that the square root in (5.4) vanishes at $\alpha=1$ if $q_{-1,-1}+q_{0,-1}+q_{1,-1}=q_{-1,1}$ and thus the (left) derivative of this square root at $\alpha=1$ is $-\infty$. Consequently, in this case the derivative of $R H$ at $\alpha=1$ is $+\infty$, so Condition A trivially holds.

Combining all these results we can formulate:

Lemma 5.4 Suppose that the following two conditions hold:

(i) $h_{1,1}+h_{0,1}+h_{1,0}>0$;

(ii) if $q_{-1,-1}+q_{0,1}+q_{1,-1}>q_{-1,1}$, then Condition $A$ is satisfied;

then there exists a unique root on $(0,1)$ of equation (5.5). If condition (i) or (ii) does not hold, then there exists no root on $(0,1)$ of equation (5.5). 
In order to complete the proof of Theorem 5.2, we now only have to show that in the case $h_{1,1}>0$ the possible second root indeed exists and lies in the interval $(-1,0)$ if condition (ii) of Lemma 5.4 holds. In this case $R H\left(0^{-}\right)=-\infty$ (compare Figure 3), so it suffices to prove, since $R H$ and $L H$ are continuous on the interval $[-1,0)$, that there exists some $\alpha \in[-1,0)$ with $R H(\alpha)>L H(\alpha)$. This proceeds rather straightforwardly now and is omitted bere.

The final aspect of this section is the interpretation of Condition $A$ as a mean drift condition. Condition (ii) in Lemma 5.4 states that if the rate downwards exceeds the rate upwards, then the inequality $R H^{\prime}>L H^{\prime}(1)$ must hold. We will show that the inequality $R H^{\prime}>L H^{\prime}(1)$ corresponds to Neuts' mean drift condition ([22], Theorem 1.7.1).

Consider a Markov process with generator $Q$ of the form

$$
Q=\left(\begin{array}{cccccc}
B_{1} & B_{0} & 0 & 0 & 0 & \ldots \\
A_{2} & A_{1} & A_{0} & 0 & 0 & \ldots \\
0 & A_{2} & A_{1} & A_{0} & 0 & \ldots \\
0 & 0 & A_{2} & A_{1} & A_{0} & \ldots \\
\vdots & \vdots & \vdots & \vdots & \vdots & \ddots
\end{array}\right)
$$

where all elements are (finite) $(k+1) \times(k+1)$ matrices. The states are denoted by $(m, n), m=0,1,2, \ldots, n=0,1, \ldots, k$ and are lexicographically ordered. The set of states $(m, 0),(m, 1), \ldots,(m, k)$ is called level $m$. Suppose that $A_{0}+A_{1}+A_{2}$ is irreducible and let $\pi$ be the solution of

$$
\pi\left(A_{0}+A_{1}+A_{2}\right)=0, \quad \pi e=1,
$$

where $e$ is the column vector with all its elements equal to one. Then by Theorem 1.7.1. in Neuts' book [22] the Markov process $Q$ is ergodic if and only if

$$
\pi A_{0} e<\pi A_{2} e
$$

The left-hand side of (5.7) can be defined as the mean drift from level $m$ to $m+1$, and the right-hand side as the mean drift from level $m+1$ to $m, m>0$, where the mean is taken with respect to the distribution $\pi$. Then (5.7) states that the mean drift to the higher level should be less than the mean drift to the lower level, and therefore is called the mean drift condition.

In our case, the generator $Q$ is also of the form (5.6), but all elements are infinite matrices and level $m$ is the infinite set of states $(m, 0),(m, 1),(m, 2), \ldots$. Hence, we cannot conclude that the mean drift condition (5.7) is a necessary and sufficient for ergodicity of $Q$. However, if we can show the equivalence of (5.7) and Condition A, then that indicates that Condition $A$ in Theorem 5.2 is not an essential extra restriction which might be removed with some effort. Let us consider therefore the mean drift condition (5.7) in case $q_{1,-1}+q_{0,-1}+q_{-1,-1}>q_{-1,1}$ (so $X_{-}(1)<1$ ) then the row vector $\pi=\left(\pi_{0}, \pi_{1}, \ldots\right)$ is given by

$$
\begin{aligned}
\pi_{n} & =C \frac{q_{-1,1}}{h_{-1,1}+h_{0,1}+h_{1,1}} & & \text { if } n=0, \\
& =C X_{-}^{n}(1) & & \text { if } n>0,
\end{aligned}
$$


where $C$ is the normalizing constant. The mean drift condition (5.7) then becomes

$$
\begin{aligned}
& \frac{X_{-}(1)}{1-X_{-}(1)} q_{1,-1}+\frac{q_{-1,1}}{h_{-1,1}+h_{0,1}+h_{1,1}}\left(h_{1,1}+h_{1,0}\right)< \\
& \frac{X_{-}(1)}{1-X_{-}(1)}\left(q_{-1,1}+q_{-1,0}+q_{-1,-1}\right)+\frac{q_{-1,1}}{h_{-1,1}+h_{0,1}+h_{1,1}}\left(h_{-1,0}+h_{-1,1}\right) .
\end{aligned}
$$

The interesting point is that the inequality $R H^{\prime}(1)>L H^{\prime}(1)$ can be rewritten as (5.8), which follows by first inserting the formula for $X_{+}(\alpha) X_{-}(\alpha)$ into equation (5.5)

$$
\frac{1}{X_{+}(\alpha)}=\frac{\left(\alpha^{2} q_{-1,-1}+\alpha q_{0,-1}+q_{1,-1}\right) X_{-}(\alpha)}{\alpha^{2} q_{-1,1}},
$$

and then, after differentiating equation (5.5), inserting the identity

$$
X_{-}^{\prime}(1)=X_{-}(1)+\frac{X_{-}(1) q_{-1,0}}{q_{1,-1}+q_{0,-1}+q_{-1,-1}-q_{-1,1}}+\frac{X_{-}^{2}(1)\left(q_{0,-1}+2 q_{-1,-1}\right)}{q_{1,-1}+q_{0,-1}+q_{-1,-1}-q_{-1,1}},
$$

which can be derived from straightforward calculations.

Summarizing, we may conclude:

Theorem 5.3 If $q_{1,-1}+q_{0,-1}+q_{-1,-1}>q_{-1,1}$, then Condition $A$ is equivalent with Neuts' mean drift condition.

\section{The finishing touch}

In this section we will apply the results of the preceding sections for the derivation of the conditions under which the compensation approach really works. In order to do this, we first investigate under what conditions the construction of formal solutions with feasible initial pairs does not fail because of degenerating of the coefficients involved. Then we will prove that the formal solutions constitute absolute convergent series, except perhaps in a neighborhood around the origin. Finally, the results obtained are summarized in the main result of this paper.

In the previous sections we derived necessary and sufficient conditions for the existence of feasible pairs $\left(\alpha_{0}, \beta_{0}\right)$ with which $x_{m, n}\left(\alpha_{0}, \beta_{0}\right)$ can be initialized so as to satisfy the convergence requirements. Let us consider those feasible pairs which fit the horizontal boundary condition (i.e. $d_{-1}=0$ and $\left|\alpha_{0}\right|>\left|\beta_{0}\right|$ ). There can at most be two such pairs and we denote them as follows if they exist:

$$
\left(\alpha_{+}, X_{-}\left(\alpha_{+}\right)\right) \text {and }\left(\alpha_{-}, X_{-}\left(\alpha_{-}\right)\right),
$$

where $\alpha_{+}$is the unique root on $(0,1)$ of equation $(5.5)$, and $\alpha_{-}$is its unique root on $(-1,0)$.

Each formal solution $x_{m, n}\left(\alpha_{0}, \beta_{0}\right)$ has its own sequence $\left\{\alpha_{i}, \beta_{i}\right\}$, which depends on the initial values $\alpha_{0}$ and $\beta_{0}$, and its own associated sequence of coefficients $\left\{c_{i}, d_{i}, e_{i}, f_{i}\right\}$ (see Section 3). For $\left(\alpha_{0}, \beta_{0}\right)=\left(\alpha_{+}, X_{-}\left(\alpha_{+}\right)\right)$, it holds that $d_{-1}=0$ and thus $d_{i}=f_{i}=0$ for all $i<0$. Hence the left branch of compensation terms vanishes. For $\alpha_{0}=\alpha_{+}$ and $\beta_{0}=X_{-}\left(\alpha_{+}\right)$we may abbreviate the notation $x_{m, n}\left(\alpha_{0}, \beta_{0}\right)$ to $x_{m, n}\left(\alpha_{+}\right)$and similar 
abbreviations may be used for other feasible pairs. So the series $x_{m, n}\left(\alpha_{+}\right)$simplifies for $m>0$ and $n>0$ to (see (3.7) and (3.8))

$$
\begin{aligned}
& x_{m, n}\left(\alpha_{+}\right)=\sum_{i=0}^{\infty} d_{i}\left(c_{i} \alpha_{i}^{m}+c_{i+1} \alpha_{i+1}^{m}\right) \beta_{i}^{n} \\
& x_{m, n}\left(\alpha_{+}\right)=d_{0} c_{0} \beta_{0}^{n} \alpha_{0}^{m}+\sum_{i=0}^{\infty} c_{i+1}\left(d_{i} \beta_{i}^{n}+d_{i+1} \beta_{i+1}^{n} \alpha_{i+1}\right) \alpha_{i+1}^{m},
\end{aligned}
$$

and on the axes to (see (3.9) and (3.10))

$$
\begin{gathered}
x_{0, n}\left(\alpha_{+}\right)=\sum_{i=0}^{\infty} e_{i} \beta_{i}^{n}, \\
x_{m, 0}\left(\alpha_{+}\right)=f_{0} \alpha_{0}^{m}+\sum_{i=0}^{\infty} f_{i+1} \alpha_{i+1}^{m},
\end{gathered}
$$

where the sequence $\left\{\alpha_{i}, \beta_{i}\right\}$ is initialized by

$$
\alpha_{0}=\alpha_{+}, \quad \beta_{0}=X_{-}\left(\alpha_{+}\right) .
$$

The solution $x_{m, n}\left(\alpha_{-}\right)$simplifies accordingly.

We will investigate now whether the construction of $x_{m, n}\left(\alpha_{+}\right)$can possibly fail because of a vanishing denominator in the definitions of the coefficients $c_{i+1}, e_{i}, d_{i+1}, f_{i+1}$ (c.f. (3.11), (3.12)).

By inserting $\beta_{i}=X_{+}\left(\alpha_{i+1}\right)$ into the common denominator in the definitions of $d_{i+1}$ and $f_{i+1}$ (formula (3.12)), we see that this denominator exactly vanishes if $\alpha_{i+1}$ solves equation (5.5). Lemma 5.2 implies

$$
\alpha_{0}>\beta_{0}>\alpha_{1}>\beta_{1}>\cdots>0
$$

and therefore only the possibility $\alpha_{i+1}=\alpha_{+}$is relevant here. However, from the monotonicity it is clear that $\alpha_{0}=\alpha_{+}$implies $\alpha_{i+1}<\alpha_{+}$.

In the same way, by inserting $\alpha_{i}=Y_{+}\left(\beta_{i}\right)$ (cf. Lemma 5.2) into the common denominator in the definitions of $c_{i+1}$ and $e_{i}$ (formula (3.11)), we see that this denominator exactly vanishes if $\beta_{i}$ solves the $\beta$-equivalent of equation (5.5). Consistingly with $\alpha_{ \pm}$the solutions of this $\beta$-equivalent might be denoted by $\beta_{+}$and $\beta_{-}$where only the possibility $\beta_{i}=\beta_{+}$ is relevant there. So, the only remaining possibility on degeneration of the coefficients is that the denominator of (3.11) vanishes if $\beta_{i}=\beta_{+}$, which really may occur, as simple examples show. This leads to the following condition (part (ii) is related to $x_{m, n}\left(\alpha_{-}\right)$):

\section{Condition}

(i) If the roots $\alpha_{+}$and $\beta_{+}$exist, then none of the $\beta_{i}$ of the sequence $\left\{\alpha_{i}, \beta_{i}\right\}_{i=0}^{\infty}$ with initial values $\alpha_{0}=\alpha_{+}$and $\beta_{0}=X_{-}\left(\alpha_{+}\right)$may be equal to $\beta_{+}$;

(ii) The same formulation as (i) with $\alpha_{+}, \beta_{+}$replaced by $\alpha_{-}, \beta_{-}$.

By considering small perturbations of the boundary probabilities, it may be shown that violation of the conditions is exceptional. In fact, in Section 7 it will be argued by using a sequence of perturbed processes, that in the case of violation of the Condition the equilibrium probabilities may be obtained by a limiting procedure. 
In the sequel of this section we suppose that the Condition and the symmetrical one with respect to the sequences $\left\{\alpha_{i}, \beta_{i}\right\}_{i=0}^{-\infty}$ associated with the series $x_{m, n}\left(\beta_{+}\right)$and $x_{m, n}\left(\beta_{-}\right)$ are satisfied.

We now try to prove that the series (6.1) converges absolutely. We need absolute convergence to guarantee that rearranging of terms is feasible (see (6.2)). The series (6.1) however, possibly diverges in states near the origin of the state space, but we will prove:

Theorem 6.1 There exists an integer $N$, such that for all feasible pairs $\left(\alpha_{0}, \beta_{0}\right)$ the following holds:

(i) The series $\sum_{i=-\infty}^{\infty} d_{i} c_{i} \alpha_{i}^{m} \beta_{i}^{m}$ and $\sum_{i=-\infty}^{\infty} d_{i} c_{i+1} \alpha_{i+1}^{m} \beta_{i}^{m}$, which add up to $x_{m, n}\left(\alpha_{0}, \beta_{0}\right)$ by definition for $m>0, n>0$, converge absolutely for all $m \geq 0, n \geq 0$ with $m+n>N$

(ii) The series $\sum_{i=-\infty}^{\infty} e_{i} \beta_{i}^{n}$, which defines $x_{0, n}\left(\alpha_{0}, \beta_{0}\right)$ for $n>0$, converges absolutely for

$$
\begin{array}{ll}
n>N-2, & \text { if } \nu_{1,1}>0 ; \\
n>N-1, & \text { if } \nu_{1,1}=0, \nu_{1,0}+\nu_{0,1}>0 ; \\
n>N, & \text { if } \nu_{1,1}=\nu_{1,0}=\nu_{0,1}=0 ;
\end{array}
$$

(iii) The series $\sum_{i=-\infty}^{\infty} f_{i+1} \alpha_{i+1}^{n}$, which defines $x_{m, 0}\left(\alpha_{0}, \beta_{0}\right)$ for $m>0$, converges absolutely for

$$
\begin{array}{ll}
m>N-2, & \text { if } h_{1,1}>0 ; \\
m>N-1, & \text { if } h_{1,1}=0, h_{1,0}+h_{0,1}>0 ; \\
m>N, & \text { if } h_{1,1}=h_{1,0}=h_{0,1}=0 ;
\end{array}
$$

(iv) $\sum_{\substack{m \geq 0, n \geq 0 \\ m+n>N}}\left|x_{m, n}\left(\alpha_{0}, \beta_{0}\right)\right|<\infty$.

We only prove Theorem 6.1 for $\left(\alpha_{0}, \beta_{0}\right)=\left(\alpha_{+}, X_{-}\left(\alpha_{+}\right)\right)$. The proof is similar for the other potential feasible initial pairs. In two lemmas we will first formulate the limit behaviour of the sequence $\left(\alpha_{i}, \beta_{i}\right)_{i=0}^{\infty}$ and the associated sequence of coefficients $\left\{c_{i}, d_{i}, e_{i}, f_{i}\right\}_{i=0}^{\infty}$. With these results the proof of the theorem itself appears to be simple.

Lemma 6.1 Let $\left|y_{1}\right|>\left|y_{2}\right|$ be the roots of (3.1) for fixed $\alpha$ with $0<|\alpha|<1$ and let

$$
z_{m, n}= \begin{cases}\alpha^{m} y_{1}^{n}+d \alpha^{m} y_{2}^{m}, & m>0, n>0 ; \\ f \alpha^{m}, & m>0, n=0,\end{cases}
$$

where $d$ and $f$ satisfy (9.5) and (9.6). Then, as $\alpha \rightarrow 0$,

$$
\begin{aligned}
& \frac{\alpha}{y_{1}} \rightarrow A_{1}, \quad \text { where } A_{1}=\frac{q-\sqrt{q^{2}-4 q_{1,-1} q_{-1,1}}}{2 q_{-1,1}} ; \\
& \frac{y_{2}}{\alpha} \rightarrow \frac{1}{A_{2}}, \quad \text { where } A_{2}=\frac{q+\sqrt{q^{2}-4 q_{1,-1} q_{-1,1}}}{2 q_{-1,1}} ; \\
& d \rightarrow-H=- \begin{cases}\frac{A_{2}}{A_{1}} & \text { if } h_{1,1}>0 ; \\
\frac{A_{2} h_{0,1}+h_{1,0}}{A_{1} h_{0,1}+h_{1,0}} & \text { if } h_{1,1}=0, h_{0,1}+h_{1,0}>0 ; \\
\frac{A_{2} h_{-1,1}-h}{A_{1} h_{1,1}-h} & \text { if } h_{1,-1}=h_{0,1}=h_{1,0}=0 ;\end{cases}
\end{aligned}
$$




$$
\begin{aligned}
& f \alpha^{-2} \rightarrow-\frac{q_{-1,1}\left(A_{2}-A_{1}\right)}{A_{1} H_{1,1}}, \quad \text { if } h_{1,1}>0 \text {; } \\
& f \alpha^{-1} \rightarrow-\frac{q_{-1,1}\left(A_{2}-A_{1}\right)}{A_{1} h_{0,1}+h_{1,0}}, \quad \text { if } h_{1,1}=0, h_{0,1}+h_{1,0}>0 \text {; } \\
& f \rightarrow-\frac{q_{-1,1}\left(A_{2}-A_{1}\right)}{A_{1} h_{-1,1}-h}, \quad \text { if } h_{1,1}=h_{0,1}=h_{1,0}=0 .
\end{aligned}
$$

Proof Rewrite equation (3.1) as an equation in $z=\beta / \alpha$. Let $z(\alpha)$ be the smaller root of that quadratic equation, so $y_{2} / \alpha=z(\alpha)$. Hence, since $z(\alpha)$ is continuous, $\alpha \rightarrow 0$,

$$
\frac{y_{2}}{\alpha}=z(\alpha) \rightarrow z(0)=\frac{1}{A_{2}},
$$

and by using the formula for the product of the two roots of a quadratic equation:

$$
\frac{\alpha}{y_{1}}=\frac{\alpha^{2} q_{-1,-1}+\alpha q_{0,-1}+q_{1,-1}}{q_{-1,1}} \frac{y_{2}}{\alpha} \rightarrow \frac{q_{1,-1}}{q_{-1,1}} \frac{1}{A_{2}}=A_{1} .
$$

The limiting behaviour of the coefficients $d$ and $f$ can be directly obtained by letting $\alpha \rightarrow 0$ in (3.5) and (3.6) and inserting (6.5) and (6.6).

By symmetry, we get the analogous result for the roots $x_{1}$ and $x_{2}$ with $\left|x_{1}\right|>\left|x_{2}\right|$ of (3.1) for fixed $\beta$. $d$ and $f$ have now to be replaced by $c$ and $e$ according to (3.3) and (3.4). The limit of $c$ when $\beta \rightarrow 0$ is then called $-V$ and is defined in the same way as $H$, with replacement of $h_{k l}$ by $v_{l k}, A_{1}$ by $1 / A_{2}$ and $A_{2}$ by $1 / A_{1}$. Applying the same substitution to the limits of $f$ (and replacing $q_{-1,1}$ by $q_{1,-1}$ ) yields the analogous limits for $e$.

If $1>\alpha_{0}>\beta_{0}>0$, which is the case we consider in the proof of Theorem 6.1 , then Lemma 4.3 implies that $\alpha_{i}$ and $\beta_{i}$ tend to zero for $i \rightarrow \infty$. Hence we directly obtain the desired limiting behaviour from Lemma 6.1 (together with its analogous version for $\beta \rightarrow 0)$ and the definitions of $\alpha_{i}, \beta_{i}, c_{i}, d_{i}, e_{i}$ and $f_{i}$ in Section 3. Note that the ratios $\beta_{i} / \alpha_{i}$ and $\alpha_{i+1} / \beta_{i}$ are monotonously decreasing, which follows from $1>\alpha_{0}>\beta_{0}>0$ with Lemma 5.2 and, particularly, Lemma 5.1. Furthermore, the following lemma is formulated for $\alpha_{0}=\alpha_{+}$in which case $h_{1,1}+h_{1,0}+h_{0,1}>0$ by Lemma 5.4 and thus for the limit of $f_{i+1}$ the case $h_{1,1}=h_{1,0}=h_{0,1}$ is not relevant (cf. Lemma 6.1).

Lemma 6.2 Consider the feasible initial pair $\alpha_{0}=\alpha_{+}, \beta_{0}=X_{-}\left(\alpha_{+}\right)$and let $i$ tend to infinity. Then we have:

As $i \rightarrow \infty$, then

$$
\frac{\beta_{i}}{\alpha_{i}} \rightarrow \frac{1}{A_{2}}, \quad \frac{\alpha_{i+1}}{\beta_{i}} \rightarrow A_{1} .
$$

If $c_{i}=0$ for some $i>0$, then $c_{j}=e_{j}=0$ for $j>i$. Otherwise, as $i \rightarrow \infty$, then

$$
\begin{aligned}
& \frac{c_{i+1}}{c_{i}} \rightarrow-V ; \\
& \frac{d_{i+1}}{c_{i}} \rightarrow-H ; \\
& \frac{e_{i}}{c_{i} \beta_{i}^{2}} \rightarrow-\frac{q_{1,-1}\left(\frac{1}{A_{1}}-\frac{1}{A_{2}}\right)}{\frac{\nu_{1,1}}{A_{2}}} \quad \text { if } \nu_{1,1}>0 ;
\end{aligned}
$$




$$
\begin{aligned}
& \frac{e_{i}}{c_{i} \beta_{i}} \rightarrow-\frac{q_{1,-1}\left(\frac{1}{A_{1}}-\frac{1}{A_{2}}\right)}{\frac{\nu_{1,0}}{A_{2}}+\nu_{0,1}} \quad \text { if } \nu_{1,1}=0, \nu_{1,0}+\nu_{0,1}>0 \text {; } \\
& \frac{e_{i}}{c_{i}} \rightarrow-\frac{q_{1,-1}\left(\frac{1}{A_{1}}-\frac{1}{A_{2}}\right)}{\frac{\nu_{1,-1}}{A_{2}}-\nu} \quad \text { if } \nu_{1,1}=\nu_{1,0}=\nu_{0,1}=0 ;
\end{aligned}
$$

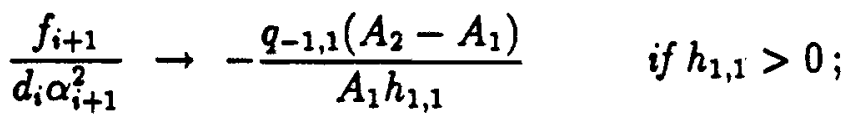

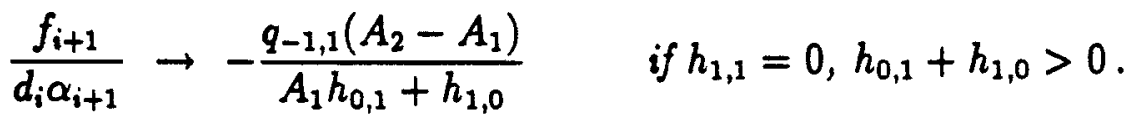

Proof of Theorem 6.1 Theorem 6.1 is trivial if $c_{i}=0$ for some $i>0$, since then $c_{j}=e_{j}=0$ for all $j>i$, and the series $x_{m, n}\left(\alpha_{+}\right)$thus simplifies to a finite sum. Now suppose that $c_{i}$ never vanishes. To prove Theorem 6.1 in this case, consider a fixed $m>0$ and $n>0$. Then by Lemma 6.2 , as $i \rightarrow \infty$,

$$
\frac{\left|d_{i+1} c_{i+1} \alpha_{i+1}^{m} \beta_{i+1}^{n}\right|}{\left|d_{i} c_{i} \alpha_{i}^{m} \beta_{i}^{n}\right|} \text { and } \frac{\left|d_{i+1} c_{i+1} \alpha_{i+2}^{m} \beta_{i+1}^{n}\right|}{\left|d_{i} c_{i+1} \alpha_{i+1}^{m} \beta_{i}^{n}\right|} \rightarrow|H V|\left(\frac{A_{1}}{A_{2}}\right)^{m+n} \text {. }
$$

Hence, if $|H V|\left(\frac{A_{1}}{A_{2}}\right)^{m+n}<1$, then the series $x_{m, n}\left(\alpha_{+}\right)$converges absolutely and if the limit $|H V|\left(\frac{A_{1}}{A_{2}}\right)^{m+n}>1$, then the series $x_{m, n}\left(\alpha_{+}\right)$diverges. Finally, if it holds that the limit $|H V|\left(\frac{A_{1}}{A_{2}}\right)^{m+n}=1$, then nothing can be said in general.

Similarly, by Lemma 6.2 , we obtain for fixed values $m>0$ and $n>0$ that as $i \rightarrow \infty$,

$$
\begin{aligned}
& \frac{\left|d_{i+1} e_{i+1} \beta_{i+1}^{n}\right|}{\left|d_{i} e_{i} \beta_{i}^{n}\right|} \rightarrow \begin{cases}|H V|\left(\frac{A_{1}}{A_{2}}\right)^{n+2} & \text { if } \nu_{11}>0 ; \\
|H V|\left(\frac{A_{1}}{A_{2}}\right)^{n+1} & \text { if } \nu_{1,1}=0, \nu_{1,0}=\nu_{0,1}>0 \\
|H V|\left(\frac{A_{1}}{A_{2}}\right)^{n} & \text { if } \nu_{1,1}=\nu_{1,0}=\nu_{0,1}=0\end{cases} \\
& \frac{\left|c_{i+2} f_{i+2} \alpha_{i+2}^{m}\right|}{\left|c_{i+1} f_{i+1} \alpha_{i+1}^{m}\right|} \rightarrow \begin{cases}|H V|\left(\frac{A_{1}}{A_{2}}\right)^{m+2} & \text { if } h_{1,1}>0 ; \\
|H V|\left(\frac{A_{1}}{A_{2}}\right)^{m+1} & \text { if } h_{1,1}=0, h_{0,1}+h_{1,0}>0\end{cases}
\end{aligned}
$$

Because $0<A_{1}<1$ and $A_{2}>1$, we can define $N$, mentioned in Theorem 6.1 as the smallest nonnegative integer such that $|H V|\left(\frac{A_{1}}{A_{2}}\right)^{N+1}<1$.

It can be directly seen from this definition and the limits (6.7) and (6.8) that $N$ is the smallest integer which guarantees absolute convergence stated in Theorem 6.1(i)-(iii). We finally prove Theorem 6.1 (iv). Inserting the series (6.1)-(6.4) yields

$$
\begin{array}{r}
\sum_{\substack{m \geq 0, n>0 \\
m+n>N}}\left|x_{m, n}\left(\alpha_{+}\right)\right|=\sum_{m=1}^{N} \sum_{n=N+1-m}^{\infty}\left|x_{m, n}\left(\alpha_{+}\right)\right|+\sum_{m=N+1}^{\infty} \sum_{n=1}^{\infty}\left|x_{m, n}\left(\alpha_{+}\right)\right|+ \\
\sum_{n=N+2}^{\infty}\left|x_{0, n}\left(\alpha_{+}\right)\right|+\sum_{m=N+2}^{\infty}\left|x_{m, 0}\left(\alpha_{+}\right)\right|
\end{array}
$$




$$
\begin{gathered}
\leq \sum_{m=1}^{N} \sum_{n=N+1-m}^{\infty} \sum_{i=0}^{\infty}\left|d_{i}\right|\left(\left|c_{i}\right| \alpha_{i}^{m}+\left|c_{i+1}\right| \alpha_{i+1}^{m}\right) \beta_{i}^{n}+ \\
\sum_{m=N+1}^{\infty} \sum_{n=1}^{\infty} \sum_{i=0}^{\infty}\left|d_{i}\right|\left(\left|c_{i}\right| \alpha_{i}^{m}+\left|c_{i+1}\right|\left(\alpha_{i}^{m}\right) \beta_{i}^{n}\right)+ \\
\sum_{n=N+2}^{\infty} \sum_{i=0}^{\infty}\left|d_{i} e_{i}\right| \beta_{i}^{n}+\sum_{m=N+2}^{\infty} \sum_{i=-1}^{\infty}\left|c_{i+1} f_{i+1}\right| \alpha_{i+1}^{m} \\
=\sum_{m=1}^{N} \sum_{i=0}^{\infty}\left|d_{i}\right|\left(\left|c_{i}\right| \alpha_{i}^{m}+\left|c_{i+1}\right| \alpha_{i+1}^{m}\right) \frac{\beta_{i}^{N+1-m}}{1-\beta_{i}}+ \\
\sum_{i=0}^{\infty}\left|d_{i}\right|\left(\left|c_{i}\right| \frac{\alpha_{i}^{N+1}}{1-\alpha_{i}}+\left|c_{i+1}\right| \frac{\alpha_{i+1}^{N+1}}{1-\alpha_{i+1}}\right) \frac{\beta_{i}}{1-\beta_{i}}+ \\
\sum_{i=0}^{\infty}\left|d_{i} e_{i}\right| \frac{\beta^{N+2}}{1-\beta_{i}}+\sum_{i=-1}^{\infty}\left|c_{i+1} f_{i+1}\right| \frac{\alpha_{i+1}^{N+2}}{1-\alpha_{i+1}}
\end{gathered}
$$

since the ratio of successive terms in these series tends to $|H V|\left(\frac{A_{1}}{A_{2}}\right)^{N+1}<1$ as $i$ tends to infinity (whereas for the last two series the limit of that ratio is possibly smaller). This completes the proof of Theorem 6.1.

$N$ may be zero, as is the case for the symmetric shortest queue problem (cf. [3]), but $N$ can also be arbitrary large, which depends essentially on the behaviour of the Markov process in the interior of the state space.

Before summarizing the results, we state one other property of the solutions obtained so far:

Lemma 6.3 The solutions $x_{m, n}\left(\alpha_{0}, \beta_{0}\right)$, for different feasible pairs $\left(\alpha_{0}, \beta_{0}\right)$ are linearly independent on the set of states $\{(m, n) \mid m, n \geq 0, m+n>N\}$, where $N$ has been defined in Theorem 6.1.

The proof is straightforward and exploits the fact that for each feasible pair $\alpha_{0}, \beta_{0}$ the first term $\alpha_{0}^{m} \beta_{0}^{n}$ in the series $x_{m, n}\left(\alpha_{0}, \beta_{0}\right)$ is dominating as $m \rightarrow \infty$ and fixed $n>0$. An alternative proof exploits Cauchy's theorem.

We now have all ingredients to prove our main result, stating that under certain drift conditions, the probabilities $p_{m, n}$ can be expressed as a linear combination of the series of products $x_{m, n}\left(\alpha_{0}, \beta_{0}\right)$, with $\left(\alpha_{0}, \beta_{0}\right)$ running through the set of at most four feasible initial pairs, on a subset of the state space. Essentially, that subset is the set on which the series $x_{m, n}\left(\alpha_{0}, \beta_{0}\right)$ converge absolutely. By Theorem 6.1 this set is given by

$$
\mathcal{A}(N)=\{(m, n) ; m \geq 0, n \geq 0, m+n>N\} \cup \mathcal{B}(N),
$$

where the set $B(N)$ depends on the specific transition structure on the vertical and horizontal axis, that is,

$$
\begin{aligned}
\mathcal{B}(N) & = \begin{cases}\{(N-1,0),(N, 0)\} & \text { if } h_{1,1}>0 ; \\
\{(N, 0)\} & \text { if } h_{1,1}=0, h_{0,1}+h_{1,0}>0 ; \\
0 & \text { if } h_{1,1}=h_{0,1}=h_{1,0}=0 ;\end{cases} \\
& \cup \begin{cases}\{(0, N-1),(0, N)\} & \text { if } \nu_{1,1}>0 ; \\
\{(0, N)\} & \text { if } \nu_{1,1}=0, \nu_{1,0}+\nu_{0,1}>0 ; \\
0 & \text { if } \nu_{1,1}=\nu_{1,0}=\nu_{0,1}=0\end{cases}
\end{aligned}
$$




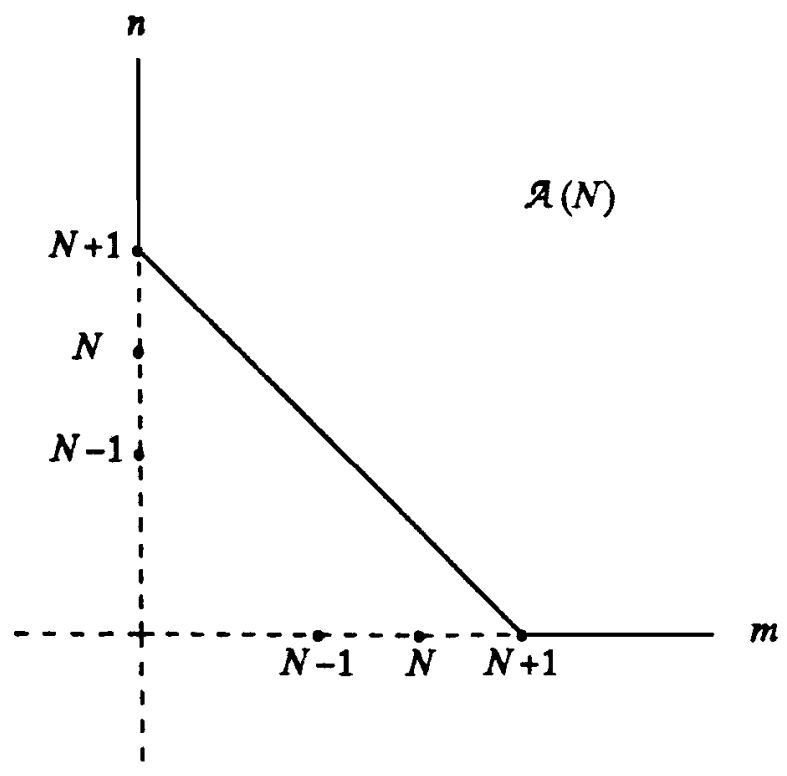

Figure 4: The set $\mathcal{A}(N)$ on which the series $x_{m, n}\left(\alpha_{0}, \beta_{0}\right)$ converge absolutely, for the special case that $h_{1,1}>0$ and $\nu_{1,1}>0$.

In Figure 4 the set $\mathcal{A}(N)$ is depicted for the special case that $h_{1,1}>0$ and $\nu_{1,1}>0$.

Theorem 6.2 (Main result) There exists an integer $M$, such that on the set $\mathcal{A}(M)$

$$
p_{m, n}=\sum_{\left(\alpha_{0}, \beta_{0}\right)} k\left(\alpha_{0}, \beta_{0}\right) x_{m, n}\left(\alpha_{0}, \beta_{0}\right)
$$

where $\left(\alpha_{0}, \beta_{0}\right)$ runs through the set of at most four feasible initial pairs and $k\left(\alpha_{0}, \beta_{0}\right)$ is some appropriately chosen coefficient, if the following conditions are satisfied:

(i) if $h_{0,1}+h_{1,1}+h_{1,0}>0$ and $q_{-1,-1}+q_{0,-1}+q_{1,-1}>q_{-1,1}$ then Condition $A$ should be satisfied

(ii) if $\nu_{1,0}+\nu_{1,1}+\nu_{0,1}>0$ and $q_{-1,-1}+q_{-1,0}+q_{-1,1}>q_{1,-1}$ then the analogon of drift Condition $A$ should hold on the vertical axis.

Proof Take $M$ at least equal to $N$, and strictly larger than 1 . The latter inequality is only required to initially exclude possible complications in the states $(0,0),(0,1),(1,1)$ and $(1,0)$, which would be due to the rates $r_{i j}$ in the origin. Then, to prove the main theorem, we shall consider the Markov process restricted to the set $\mathcal{A}(M)$, that is, visits to states outside $\mathcal{A}(M)$ are not considered.

In all states with $m+n>M$ the equilibrium equations, associated with the restricted process, are identical to the ones of the original process, that is, the equations $(2.1)-(2.5)$. Hence, for each feasible pair $\left(\alpha_{0}, \beta_{0}\right)$ the series $x_{m, n}\left(\alpha_{0}, \beta_{0}\right)$, which converges absolutely on the set $\mathcal{A}(M)$, satisfies the equilibrium equations associated with the restricted process in all states $m+n>M$. The boundary equations on $\mathcal{B}(M)$ are not given by the equations (2.3) and (2.5), but have an extra incoming rate. This is due to excursions of the original process to states outside $\mathcal{A}(M)$, which because of the special transition structure in the interior of the state space, always end at one of the states in $\mathcal{B}(M)$. To finally satisfy the equations on $\mathcal{B}(M)$, we will try to fit a linear combination of series $x_{m, n}\left(\alpha_{0}, \beta_{0}\right)$, with different pairs $\left(\alpha_{0}, \beta_{0}\right)$, on these equations.

Since the original Markov process is supposed to be irreducible (see Section 2), it follows that $h_{0,1}+h_{1,1}+h_{1,0}$ or $\nu_{1,0}+\nu_{1,1}+\nu_{0,1}$ is positive. The conditions (i) and (ii) 
in Theorem 6.2 are necessary and sufficient for the existence of a sufficient number of feasible pairs $\left(\alpha_{0}, \beta_{0}\right)$, in the sense that the number of feasible pairs $\left(\alpha_{0}, \beta_{0}\right)$ equals the number of states in $\mathcal{B}(M)$. Hence, by first omitting one arbitrarily chosen equation on $\mathcal{B}(M)$, it follows that there exist nonnull coefficients $k\left(\alpha_{0}, \beta_{0}\right)$ such that

$$
\sum_{\left(\alpha_{0}, \beta_{0}\right)} k\left(\alpha_{0}, \beta_{0}\right) x_{m, n}\left(\alpha_{0}, \beta_{0}\right),
$$

where $\left(\alpha_{0}, \beta_{0}\right)$ runs through the set of feasible pairs, satisfies the remaining (homogeneous) equilibrium equations on $\mathcal{B}(M)$. The equation on $\mathcal{B}(M)$, which was initially omitted, is automatically satisfied. More precisely, inserting the linear combination (6.9) into the other equations on $\mathcal{A}(M)$ and then summing over these equations and changing summations exactly yields the desired equation. Changing summations is allowed by the absolute convergence stated in Theorem 6.1(iv). The linear combination (6.9) is nonnull, because, by Lemma 6.3 , the series $x_{m, n}\left(\alpha_{0}, \beta_{0}\right)$ are linearly independent on the set of states with $m+n>M$. By a result of Foster ([3], Theorem 1), this proves that the process restricted to $\mathcal{A}(M)$ is ergodic. The linear combination (6.9) can be normalized to produce the equilibrium distribution $\left\{p_{m, n}(M)\right\}$ of the process restricted to $\mathcal{A}(M)$. Since the number of states outside $\mathcal{A}(M)$ is finite, it follows that the original process is also ergodic and for all $(m, n) \in \mathcal{A}(M)$ the probabilities $p_{m, n}$ and $p_{m, n}(M)$ are related by

$$
p_{m, n}=p_{m, n}(M) P(\mathcal{A}(M)),
$$

where $P(\mathcal{A}(M))$ is the probability that the original process is in the set $\mathcal{A}(M)$. Since the linear combination (6.9) equals $p_{m, n}(M)$ up to a normalizing constant, this finally proves Theorem 6.2.

\section{Conclusions and Comments}

It has been proven in the preceding sections that the compensation approach which worked for some specific problems, can be extended to more general random walks on two-dimensional grids. For random walks on the first quadrant it appeared that the essential restriction for the method is constituted by the requirement

$$
q_{1,1}+q_{1,0}+q_{0,1}=0 \quad \text { (Assumption of Section 4). }
$$

If there is a drift in north-east direction, then the compensation procedure does not work. In some cases (like the two independent $M|M| 1$-queues) no compensation is needed, but in other cases (like the case of two coupled $M|M| 1$-queues, cf. [13] and [21]) compensation would be necessary and the solution becomes essentially more complicated.

The compensation method is a numerically oriented method and leads to efficient numerical procedures, not only for the equilibrium probabilities, but also for other processcharacteristics like the mean and variance of the waiting-time. Such procedures will not be worked out here for the general model of this paper. In papers on specific models (cf [1], [3]) the authors have worked out such procedures in more detail. It should be mentioned here that such specific models have some extra properties which can be exploited for the numerical analysis. In the symmetric shortest queue model as well as in Hofri's multiprogramming model it appeared that only the pair $\left(\alpha_{+}, X_{-}\left(\alpha_{+}\right)\right)$plays a role, so we don't get a linear combination of more series. In the ssymmetric shortest queue problem, 
which does not fit into the structure of the Markov processes treated in this paper, it appeared that two series were essential (cf. [4]).

For the symmetric shorest queue problem, $N$ was equal to zero, but, for Hofri's multiprogramming problem, $N$ could be positive, depending on the parameter values.

As said before the non-existence of a north-east drift is apparently an essential restriction for the applicability of the compensation method. Other assumptions made in this paper seem either to be natural (like the drift conditions of Section 5) or non-essential. In the sequel of this section we comment on several technical aspects of the paper, mainly regarding such seemingly non-essential but convenient restrictions.

a. The state space was chosen as the first quadrant of the two-dimensional grid. In the opinion of the authors, the extension to higher dimensions should be feasible as well as the extension to other boundary structures. The case of three dimensions is investigated currently, but as yet without definitive results.

The asymmetric shortest queue problem appears to represent a random walk on the grid of the right half plane with different properties for the upper and lower quadrant. So, the horizontal axis forms a kind of frontier between the two areas. The method appears to be well-extendable to this case (cf. [4]). Hence one might expect that extensions with regard to the form of the state space are quite well possible.

b. The restriction to random walks with transitions of at most one unit in either direction was made purely for convenience, since otherwise more states would generate nonstandard equilibrium equations. This restriction seems to be completely nonessential. However, a general treatment of the case with transitions of at most $n$ units in either direction seems to be fairly complicated. For special cases the approach seems to be tractable as appears from the next comment.

c. The restriction to purely Markovian behaviour seems to be nonessential. In [2], first attempts are described of treating $E_{k}\left|E_{r}\right| c$ queueing systems by the same approach. It appears that only finitely many terms are necessary. By considering a customer as a set of phases, the process makes larger jumps. In this way we get very special examples of the extension mentioned in $b$.

d. A special case arises when the behaviour at a boundary is just the truncation of the behaviour at interior points, i.e. $\nu_{i j}=q_{i j}$ (or $h_{j 1}=q_{j 1}$ ) for all $i$, then $e_{i}=d_{i}\left(c_{i}+c_{i+1}\right)$ and one does not need the extra degree of freedom in states $(0, n)$. So, $(3.9)$ is not needed and (3.7) also holds for $m=0$.

e. In Sections 3 and 4 assumptions were introduced requiring that for the interior points, rate components to the east, west, north, south, and south-west would be present, whereas (most essentially) no rate component to the north-east was allowed.

These assumptions exclude the special case that all $q_{i j}$, except $q_{-1,1}$ and $q_{1,-1}$ are zero. The results are essentially still valid for this special case (except when $q_{-1,1}$ and $q_{1,-1}$ are equal), and often simplify. In particular, in this case equation (3.1) further simplifies to

$$
(\alpha-\beta)\left(\alpha q_{-1,1}-\beta q_{1,-1}\right)=0,
$$

for which it is easy to prove, if $\alpha_{0}=\beta_{0}$, that for all $i$

$$
\alpha_{i}=\left(\frac{q_{1,-1}}{q_{-1,1}}\right)^{i} \alpha_{0}, \quad \beta_{i}=\left(\frac{q_{1,-1}}{q-1,1}\right)^{i} \beta_{0} .
$$


f. We will briefly discuss the special cases that are initially excluded by the assumption in Section 3. We will first treat the case that part (i) is violated, so $q_{1,1}+q_{1,0}+q_{1,-1}=0$ (there is no rate component to the east), where, to avoid trivialities, we also assume that

$$
h_{1,1}+h_{1,0}>0 \text {. }
$$

Then it can be proved under certain drift conditions, that for all $m>1$ the probabilities $p_{m, n}$ can be expressed by a linear combination of the initial products $\alpha_{0}^{m} \beta_{0}^{n}$ which can be fit on the horizontal boundary $\left(d_{-1}=0\right)$. This can be established by restricting the Markov process to the set

$$
\{(m, n), m>1, n \geq 0\} \cup\{(1,0)\}
$$

and then proceed analogously as in Section 4. To restrict the process to the above set of states we need that as soon as the process enters the set of states with $m \leq 1$, then the expected time to return to $(1,0)$ if finite, for which a necessary and sufficient condition can be derived from Neuts' mean drift condition ([21], Theorem 1.7.1).

Now we will consider the case that part (ii) is violated, so $q_{-1,1}+q_{-1,0}+q_{-1,-1}=0$ (there is no rate component to the west), where to avoid trivialities, we also assume that

$$
h_{-1,1}+h_{-1,0}>0 \text {. }
$$

This case will be treated by means of the following typical example of the longer queue model:

Consider a system consisting of two queues that are served by one server. The service times are exponentially distributed with unit mean. The server always works on the longer queue and treats the jobs in the longer queue with preemptive priority with respect to the jobs in the smaller queue. In each queue jobs arrive according to a Poisson stream with intensity $\frac{\rho}{2}$. This problem is known as the longer queue model. The state space consists of the pairs $(m, n), m, n=0,1, \ldots$ where $m$ is the length of the shorter queue and $m+n$ the length of the longer queue. Jobs in service are also counted as being in queue.

Now equation(3.1) reduces to a linear equation in $\alpha$. Therefore the generation of compensations terms fails and, due to the vertical boundary conditions, the probabilities $p_{m, n}$ cannot be expressed by a linear combination of the initial products $\alpha_{0}^{m} \beta_{0}^{n}$ which can be fit on the horizontal boundary. Instead of the compensation approach there are several other ways to solve the longer queue model. In fact, that problem has been extensively studied by Zheng and Zipkin [23] and Flatto [14], leading to the following result:

$$
p_{m, n}=\lambda^{n} P_{m}(n),
$$

where $P_{m}(n)$ is a polynomial in $n$ of degree $m$. So, the probabilities do not have the geometric form.

We have assumed that jobs in the longer queue are treated with preemptive priority with respect to the jobs in the shorter queue. The longer queue model, where jobs in the longer queue are treated with nonpreemptive priority, has been studied by Cohen [10]. He treats the case of a general service time distribution and reduces the functional equation to a Riemann type boundary value problem. 
g. The Condition of Section 6 was introduced to avoid the rather special cases that for one of the appropriate initial pairs the construction of the formal solution $x_{m, n}\left(\alpha_{0}, \beta_{0}\right)$ fails. Suppose that we have a situation where we would need and expect the solutions $x_{m, n}\left(\alpha_{+}\right)$and $x_{m, n}\left(\beta_{+}\right)$, but the construction of $x_{m, n}\left(\alpha_{+}\right)$fails, since the Condition is not satisfied. Then we perturb the behaviour on the vertical boundary (replace $\nu_{0,1}$ by $\nu_{0,1}+\varepsilon$ with $\left.\varepsilon>0\right)$ and consider the relevant solutions $x_{m, n}^{e}\left(\alpha_{+}\right)$and $x_{m, n}^{e}\left(\beta_{+}(\varepsilon)\right)$ for the perturbed process which start at $\alpha_{+}$and $\beta_{+}(\varepsilon)$, respectively. Direct application of $\varepsilon \downarrow 0$ does, of course, not give anything new, but after introduction of higher order terms in the form of derivatives with respect to $\varepsilon$ we get for $\varepsilon \downarrow 0$ extra terms in the form $m \alpha^{m} \beta^{n}$ and $n \alpha^{m} \beta^{n}$.

Finally we mention that the parts (iv) and (v) of the Assumption of Section 3 can be relaxed: it suffices if at least one of the axis is reflecting.

\section{References}

[1] I.J.B.F. Adan, G.J. van Houtum, J. Wessels, W.H.M. Zijm. A compensation procedure for multiprogramming queues. Submitted for publication.

[2] I.J.B.F. Adan, W.A. van de Waarsenburg, J. Wessels. Analysing $E_{k}\left|E_{r}\right| c$-queues. Memorandum COSOR. (1991) Technical University Eindhoven (in preparation).

[3] I.J.B.F. Adan, J. Wessels, W.H.M. Zijm. Analysis of the symmetric shortest queue problem. Stochastic Models 6 (1990) 691-713.

[4] I.J.B.F. Adan, J. Wessels, W.H.M. Zijm. Analysis of the asymmetric shortest queue problem. Queueing Systems 8 (1991) 1-58.

[5] I.J.B.F. Adan, J. Wessels, W.H.M. Zijm. Analysis of the asymmetric shortest queue problem with threshold jockeying. Stochastic Models 7 (1991) nr. 4 (to appear).

[6] I.J.B.F. Adan, J. Wessels, W.H.M. Zijm. Analysing multiprogramming queues by generating functions. Submitted for publication.

[7] F. Baskett, K.M. Chandy, R. Muntz, F. Palacios-Gomez. Open, closed and mixed networks of queues with different classes of customers. Journal of the ACM 22 (1975) 248-260.

[8] J.P.C. Blanc. On a numerical method for calculating state probabilities for queueing systems with more than one waiting line. J. Comput. Appl. Math. 20 (1987) 119-125.

[9] J.P.C. Blanc. The power-series algorithms applied to the shortest queue model. Memorandum 379. Tilburg University, Department of Economics (1989).

[10] J.W. Cohen. A two-queue, one-server model with priority for the longer queue. Queuing Systems 2 (1987) 261-283.

[11] J.W. Cohen, O.J. Boxma. Boundary value problems in queueing system analysis. North-Holland, Amsterdam (1983).

[12] G. Fayolle. Méthodes analytiques pour les files d'attente coupleés. Thesis, Univ. de Paris VI, Paris (1979). 
[13] G. Fayolle, R. Iasnogorodski. Two coupled processors: the reduction to a RiemannHilbert problem. Z. Wahrscheinlichkeitsth. Verw. Gebiete 47 (1979) 325-351.

[14] L. Flatto. The longer queue model. Probab. Engineer. Inform. Sci. 3 (1989) 537-559.

[15] L. Flatto, H.P. McKean. Two queues in parallel. Comm. Pure Appl. Math. 30 (1977) 255-263.

[16] F.G. Foster. On the stochastic matrices associated with certain queueing processes. Ann. Math. Statist. 24 (1953) 355-360.

[17] M. Hofri. A generating-function analysis of multiprogramming queues. Intern. J. Computer and Information Sciences 7 (1978) 121-155.

[18] G. Hooghiemstra, M. Keane, S. van de Ree. Power series for stationary distributions of coupled processor models. SIAM J. Appl. Math. 48 (1988) 1159-1166.

[19] R. Iasnogorodski. Problèmes-frontières dans les files d'attente. Thesis, Univ. de Paris IV, Paris (1979).

[20] J.F.C. Kingman. Two similar queues in parallel. Ann. Math. Statist. 32 (1961) 13141323.

[21] A.G. Konheim, J. Meilijson, A. Melkman. Processor-sharing of two parallel lines. J. Appl. Probab. 18 (1981) 952-956.

[22] M.F. Neuts. Matrix-geometric solutions in stochastic models. Johns Hopkins University Press, Baltimore (1981).

[23] Y.S. Zheng, P. Zipkin. A queueing model to analyze the value of centralized inventory information. Research working paper no. 86-6, Columbia University Business School, New York (1986). 
EINDHOVEN UNIVERSITY OF TECHNOLOGY

Department of Mathematics and Computing Science

PROBABILITY THEORY, STATISTICS, OPERATIONS RESEARCH

AND SYSTEMS THEORY

P.O. BOX 513

5600 MB Eindhoven, The Netherlands

Secretariate: Dommelbuilding 0.03

Telephone : 040-473130

-List of CosoR-memoranda - 1991

\begin{tabular}{|c|c|c|}
\hline Number & Month & Author \\
\hline $91-01$ & January & $\begin{array}{l}\text { M.W.I. van Kraaij } \\
\text { W.Z. Venema } \\
\text { J. Wessels }\end{array}$ \\
\hline $91-02$ & January & $\begin{array}{l}\text { M.W.I. van Kraaij } \\
\text { W.Z. Venema } \\
\text { J. Wessels }\end{array}$ \\
\hline $91-03$ & January & M.W.P. Savelsbergh \\
\hline $91-04$ & January & M.W.I. van Kraaij \\
\hline
\end{tabular}

\section{Title}

The construction of a strategy for manpower planning problems.

Support for problem formulation and evaluation in manpower planning problems.

The vehicle routing problem with time windows: minimizing route duration.

Some considerations concerning the problem interpreter of the new manpower planning system formasy.

A cutting plane algorithm for the single machine scheduling problem with release times.

Properties of FourierStieltjes sequences of distribution with support in $[0,1)$.

Analysis of a two-phase inspection model with competing risks.

The Design and Complexity of Exact Multi-Layered Perceptrons.

The Classification Capabilities of Exact

Two-Layered Peceptrons.

Sorting With A Neural Net.

P.J. Zwietering

E.H.L. Aarts

J. Wessels

91-11 May F. Coolen
On some misconceptions about subjective probability and Bayesian inference. 


\begin{tabular}{|c|c|c|}
\hline $91-12$ & May & P. van der Laan \\
\hline $91-13$ & May & $\begin{array}{l}\text { I.J.B.F. Adan } \\
\text { G.J. van Houtum } \\
\text { J. Wessels } \\
\text { W.H.M. Zijm }\end{array}$ \\
\hline $91-14$ & June & $\begin{array}{l}\text { J. Korst } \\
\text { E. Aarts } \\
\text { J.K. Lenstra } \\
\text { J. Wessels }\end{array}$ \\
\hline $91-15$ & July & $\begin{array}{l}\text { P.J. 2wietering } \\
\text { M.J.A.L. van Kraaij } \\
\text { E.H.L. Aarts } \\
\text { J. Wessels }\end{array}$ \\
\hline $91-16$ & July & $\begin{array}{l}\text { P. Deheuvels } \\
\text { J.H.J. Einmahl }\end{array}$ \\
\hline
\end{tabular}

$91-17$

$91-18$

$91-19$

$91-20$

$91-21$

$91-22$

$91-23$
August

M.W.P. Savelsbergh

G.C. Sigismondi

G.L. Nemhauser

August

M.W.P. Savelsbergh

G.C. Sigismondi

G.L. Nemhauser

August

P. van der Laan

September

P. van der Laan

September

E. Levner

A.S. Nemi rovsky

September R.J.M. Vaessens

E.H.L. Aarts

J.H. van Lint

September
Two-stage selection procedures with attention to screening.
A compensation procedure for multiprograming queues.

Periodic assignment and graph colouring.

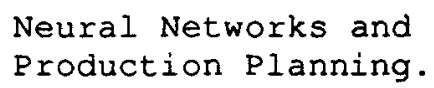

Functional description of MINTO, a Mixed INTeger Optimizer.

MINTO, a Mixed INTeger Optimizer.

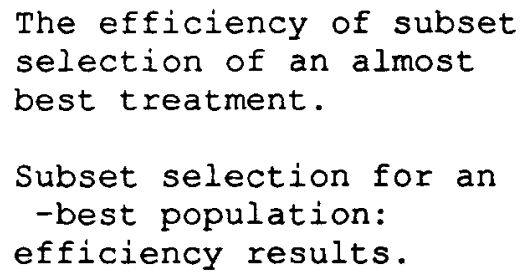

A network flow algorithm for just-in-time project scheduling.

Genetic Algorithms in Coding Theory - A Table for $A_{3}(n, d)$.

Distribution theory for selection from logistic populations. 


\begin{tabular}{|c|c|c|}
\hline $91-24$ & October & $\begin{array}{l}\text { I.J.B.F. Adan } \\
\text { J. Wessels } \\
\text { W.H.M. Zijm }\end{array}$ \\
\hline $91-25$ & October & $\begin{array}{l}\text { I.J.B.F. Adan } \\
\text { J. Wessels } \\
\text { W.H.M. Zijm }\end{array}$ \\
\hline $91-26$ & October & $\begin{array}{l}\text { E.E.M. van Berkum } \\
\text { P.M. Upperman }\end{array}$ \\
\hline $91-27$ & October & $\begin{array}{l}\text { R.P. Gilles } \\
\text { P.H.M. Ruys } \\
\text { S. Jilin }\end{array}$ \\
\hline $91-28$ & October & $\begin{array}{l}\text { I.J.B.E. Adan } \\
\text { J. Wessels } \\
\text { W.H.M. Zijm }\end{array}$ \\
\hline
\end{tabular}

Matrix-geometric analysis

of the shortest queue problem with threshold jockeying.

Analysing Multiprogramming Queues by Generating Functions.

D-optimal designs for an incomplete quadratic model.

Quasi-Networks in Social

Relational Systems.

A Compensation Approach for Two-dimensional Markov

Processes 